\title{
Enzymatic glycosylation involving fluorinated carbohydrates
}

Article

Accepted Version

Council, C. E., Kilpin, K. J., Gusthart, J. S., Allman, S. A., Linclau, B. and Lee, S. S. (2020) Enzymatic glycosylation involving fluorinated carbohydrates. Organic \& Biomolecular Chemistry, 18 (18). pp. 3423-3451. ISSN 1477-0520 doi: https://doi.org/10.1039/D0OB00436G Available at https://centaur.reading.ac.uk/90489/

It is advisable to refer to the publisher's version if you intend to cite from the work. See Guidance on citing.

Published version at: http://dx.doi.org/10.1039/D0OB00436G

To link to this article DOI: http://dx.doi.org/10.1039/D0OB00436G

Publisher: Royal Society of Chemistry

All outputs in CentAUR are protected by Intellectual Property Rights law, including copyright law. Copyright and IPR is retained by the creators or other copyright holders. Terms and conditions for use of this material are defined in the End User Agreement.

\section{www.reading.ac.uk/centaur}

\section{CentAUR}

Central Archive at the University of Reading 
Reading's research outputs online 


\section{Enzymatic glycosylation involving fluorinated carbohydrates}

Claire E. Council ${ }^{\mathrm{a}}$, Kelly J. Kilpin ${ }^{\mathrm{a}}$, Jessica S. Gusthart ${ }^{\mathrm{b}}$, Sarah A. Allman ${ }^{\mathrm{b}}$, Bruno Linclau ${ }^{\mathrm{a}}$, and Seung Seo Lee ${ }^{\mathrm{a}}$

a School of Chemistry, University of Southampton, Highfield, Southampton, SO17 1BJ, UK

${ }^{\mathrm{b}}$ Reading School of Pharmacy, University of Reading, Whiteknights, Reading, RG6 6UR, UK

\section{Abstract}

Fluorinated carbohydrates, where one (or more) fluorine atom(s) have been introduced into a carbohydrate structure, typically through deoxyfluorination chemistry, have a wide range of applications in the glycosciences. Fluorinated derivatives of galactose, glucose, $N$-acetylgalactosamine, $N$-acetylglucosamine, talose, fucose and sialic acid have been employed as either donor or acceptor substrates in glycosylation reactions. Fluorinated donors can be synthesised by synthetic methods or produced enzymatically from chemically fluorinated sugars. The latter process is mediated by enzymes such as kinases, phosphorylases and nucleotidyltransferases. Fluorinated donors produced by either method can subsequently be used in glycosylation reactions mediated by glycosyltransferases, or phosphorylases yielding fluorinated oligosaccharide or glycoconjugate products. Fluorinated acceptor substrates are typically synthesised chemically. Glycosyltransferases are most commonly used in conjunction with natural donors to further elaborate fluorinated acceptor substrates. Glycoside hydrolases are used with either fluorinated donors or acceptors. The activity of enzymes towards fluorinated sugars is often lower than towards the natural sugar substrates irrespective of donor or acceptor. This may be in part attributed to elimination of the contribution of the hydroxyl group to the binding of the substrate to enzymes. However, in many cases, enzymes still maintain a significant activity, and reactions may be optimised where necessary, enabling enzymes to be used more successfully in the production of fluorinated carbohydrates. This review describes the current state of the art regarding chemoenzymatic production of fluorinated carbohydrates, focusing specifically on examples of the enzymatic production of activated fluorinated donors and enzymatic glycosylation involving fluorinated sugars as either glycosyl donors or acceptors. 


\section{Introduction}

Fluorinated carbohydrates have been employed for a variety of applications in the fields of biochemistry, structural biology, cell biology and immunology. ${ }^{1-6}$ They have found uses as mechanistic probes for kinetic ${ }^{7,8}$ and structural studies ${ }^{9}$ to help understand protein-carbohydrate binding and catalytic mechanisms of enzymes. The in-vitro post-translational modification of proteins with fluorinated carbohydrates has been demonstrated to be an effective tool to examine the roles of glycans using ${ }^{19} \mathrm{~F} \mathrm{NMR} .{ }^{2}$ Additionally, positron emission tomography (PET) allows non-invasive and non-destructive in vivo analysis of carbohydrates incorporating ${ }^{18} \mathrm{~F}$ in biological systems. ${ }^{5}$ Approaches such as these are advantageous as they allow the specific behaviour of the fluorinated species to be analysed without competing signals arising from other components of the biological milieu. ${ }^{10,11}$ Fluorination has also been shown to endow carbohydrate structures with increased resistance towards degradative enzymes. ${ }^{12}$ This inherent stability makes fluorinated carbohydrates attractive components for glycoconjugate vaccines. ${ }^{13}$

Chemo-enzymatic approaches to the synthesis of natural and chemically modified carbohydrates are well established. The review of Li, McArthur and Chen ${ }^{14}$ provides an excellent summary of chemical and enzymatic transformations using natural and synthetic sugars. The reviews of Uhrig and Guo et al. ${ }^{15-16}$ provide overviews of synthetic strategies for fluorination of carbohydrates and methods of introducing stereoselective fluorination into monosaccharide structures. Enzymatic synthesis of fluorosugars has however not previously been the subject of a focussed extensive review. This work therefore focuses on examining the specific enzymes that accept fluorinated sugars as donors or acceptors and how such enzymes are employed to affect the transformation of larger and more complex carbohydrate structures.

The success of chemoenzymatic synthesis approaches with modified substrates relies upon the ability of the enzyme to both recognise and utilise such substrates despite changes in structure. Fluorine substitution has been shown to have only minimal effect on the conformation of sugars, ${ }^{17}$ and this has been shown to hold true even if sugars bear multiple fluorine substituents. ${ }^{18}$ However, as fluorine atoms function solely as (weak) hydrogen bond acceptors and not as a hydrogen bond donors this can influence the hydrogen bond properties of adjacent hydroxyl group. ${ }^{19,20}$ 
Some enzymes display high substrate specificity. In some cases, even a single hydroxyl to fluorine substitution can prevent a sugar from acting as a substrate. ${ }^{21}$ Others are more tolerant to fluorinated sugars albeit with lower catalytic efficiency as discussed later in this article. Field and co-workers ${ }^{22}$ suggest that even at low efficiency, enzymatic biotransformations can still provide access to biomolecules that would be laborious to prepare via chemical synthesis as enzymes become more widely available.

Enzymes which catalyse glycosylation reactions typically require an activated donor such as a sugar-1phosphate or sugar nucleotide mono- or diphosphate. The synthesis of fluorinated donor molecules can often also be achieved enzymatically, therefore enzymes which are capable of carrying out these conversions to achieve the full enzymatic conversion from fluorinated reducing sugar to glycan are also covered. It must also be noted these 'intermediates' are often interesting target molecules in their own right as many have been shown to be enzymatic inhibitors and have found applications as mechanistic probes. ${ }^{8}$

This review is organised by fluorinated sugar. For each named sugar, when and as appropriate, the enzymatic synthesis of activated sugar donors and their subsequent use in glycosylation reactions is first discussed, grouped by enzyme class, followed by glycosylation using the named sugar as acceptor, either as a monosaccharide or as part of a larger glycan, again organised by enzyme class. For the purposes of this review the nomenclature used for the fluorinated sugar derivatives does not distinguish between deoxy-fluorination and fluorination; 6FGal refers to 6-deoxy-6-fluorogalactose, whilst 6FFuc refers to fucose fluorinated at position 6. Where there is geminal difluorination, the original sugar name is maintained for clarity. Unless indicated, all sugars possess the D-configuration. 
Carbohydrate synthesis and degradation in vivo is mediated by a suite of glycoenzymes. In this article, these glycoenzymes refer to any enzymes that synthesise or modify carbohydrate substrates and are limited to those that have been used with fluorinated carbohydrates. Thus, the enzyme groups presented in this glossary are classified differently from the CAZY database (http://www.cazy.org), and the classification follows the discussion in this article. The enzyme groups presented in this glossary are: kinases, pyrophosphorylases (or nucleotidyltransferases), glycosyltransferases, glycoside hydrolases and phosphorylases. A summary of the actions of key glycoenzymes in this article is presented in Scheme 1.

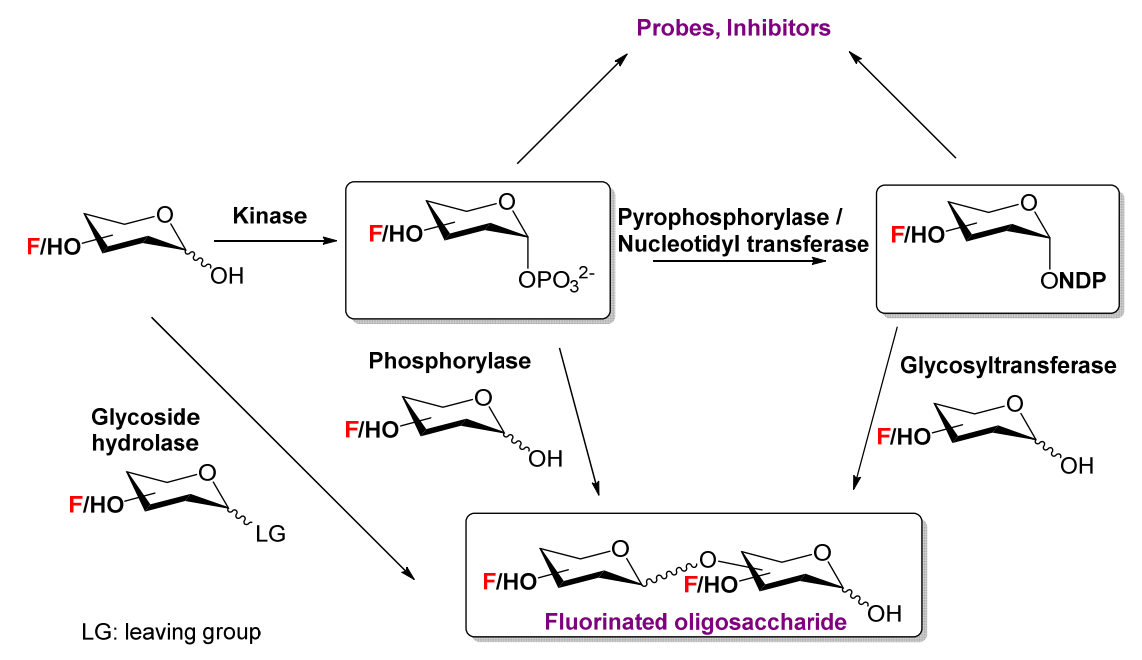

Scheme 1 Summary of key enzymes used in the formation of fluorinated donors and for enzymatic glycosylation.

\section{Kinases}

Kinases transfer a phosphate group from a high energy donor - typically adenosine triphosphate (ATP) - to the anomeric position of a reducing sugar with $\alpha$-selectivity. Galactose kinase (GalK), N-acetylgalactosamine kinase (GalNAcK) and N-acetylhexosamine kinase (NahK) transfer phosphate to the 1-position of Gal, GalNAc and $\mathrm{N}$-acetylhexoses respectively. Alternatively, hexokinase catalyses the transfer of phosphate to the 6-position of hexoses. This enzyme can be used with a phosphoglucomutase (PGM) which is capable of isomerising the phosphate group to the anomeric position, again with $\alpha$-selectivity.

Pyrophosphorylases 
Pyrophosphorylase (or nucleotidyltransferase) enzymes transfer nucleotide monophosphate (NMP) to the phosphosugar to give the nucleotide diphosphate (NDP) sugar. The mechanism involves the formation of a ternary complex where a nucleotide triphosphate (NTP) and the sugar-1P substrate successively bind into the active site. This allows nucleophilic attack by the anomeric phosphate moiety onto the first nucleotide linked phosphate group, which releases pyrophosphate and the sugar nucleotide.

\section{Glycosyltransferases}

Glycosyltransferases form a glycosylic bond between an activated sugar (usually nucleotidyl phosphate sugar) donor and a sugar acceptor such as monosaccharide, oligosaccharide or non-sugar molecule. Glycosyltransferases are named for the donor sugar they transfer, so a galactosyltransferase (GalT) transfers activated galactose onto an acceptor, a sialyltransferase (SiaT) transfers activated sialic acid etc. Generally, GalTs utilise a uridine diphosphate (UDP) donor and SiaTs utilise cytosine monophosphate (CMP) -activated sialic acids as the sialic acid donor.

\section{Glycoside hydrolases}

As defined in CAZY database (http://www.cazy.org), glycoside hydrolases catalyse hydrolysis of glycosidic bonds. Although glycoside hydrolases are naturally degradative enzymes, under certain conditions some enzymes in this class can be directed to the synthetic reaction via reverse hydrolysis or transglycosidation. ${ }^{23}$ Some glycoside hydrolases (such as the trans-sialidase from Trypanosoma cruzi) are known to have higher synthetic activity than hydrolytic activity, with the active site more suitably accommodating a non-water nucleophile such as a sugar molecule. Trans-sialidases transfer sialic acid from a donor oligosaccharide containing a terminal sialic acid, such as the heavily sialylated glycoprotein fetuin, to another Gal terminated acceptor with $\alpha-(2 \rightarrow 3)$ configuration. ${ }^{24}$ In synthetic applications, trans-sialidases have been used as an alternative to sialyltransferases to introduce sialic acid to carbohydrate structures.

\section{Phosphorylases}

Phosphorylases catalyse the reversible formation of a monosaccharide-1-phosphate from an oligosaccharide and an inorganic phosphate. The reverse reaction can be used to synthesize oligosaccharides. 


\section{Fluorinated Galactose}

Early evidence that deoxyfluorogalactose-1-phosphate sugar derivatives could be synthesised enzymatically from the corresponding deoxyfluorogalactopyranose came from work carried out by Kent and Wright. ${ }^{25}$ In this study, they demonstrated the conversion of $6 \mathrm{FGal}$ to $6 \mathrm{FGal}-1 \mathrm{P}$ using a yeast-derived galactokinase $(\mathrm{GalK})$ in the presence of ATP. Westwood et al. ${ }^{26}$ later evidenced that $2 \mathrm{FGal}, 3 \mathrm{FGal}$ and $4 \mathrm{FGal}$ could be converted by the same enzyme to 2FGal-1P, 3FGal-1P and 4FGal-1P respectively. The results of their kinetic study suggests the yeast-derived GalK has lower affinity for the four fluorinated substrates when compared with the natural Gal substrate (in the order Gal $>2 \mathrm{FGal}>3 \mathrm{FGal} \approx 6 \mathrm{FGal}<<4 \mathrm{FGal}$ ).

These early findings were later verified by other groups who exploited GalK to synthesise the anomeric phosphates 2FGal-1P, 3FGal-1P and 6FGal-1P on a larger scale. ${ }^{21-22,27}$ In these studies, a one-pot, two-enzyme system was employed, with the anomeric phosphate converted directly to the UDP-sugar by the pyrophosphorylase Gal-1P uridyltransferase (GalPUT).

Hayashi et al. ${ }^{21}$ reported that yeast GalK catalysed the synthesis of $2 \mathrm{FGal}-1 \mathrm{P}$ in preparative quantities from 2FGal (Scheme 2). Stereoselective conversion to the $\alpha$-anomer was confirmed by NMR spectroscopy. The isolated 2FGal-1P was then converted to UDP-2FGal using GalPUT, coupled with a UDP-Glc regeneration cycle, which avoids the requirement for stoichiometric quantities of commercially available but costly UDPGlc. The direct synthesis of $\alpha$-UDP-2FGal from $2 \mathrm{FGal}$ was also possible without isolating the phosphate intermediate.

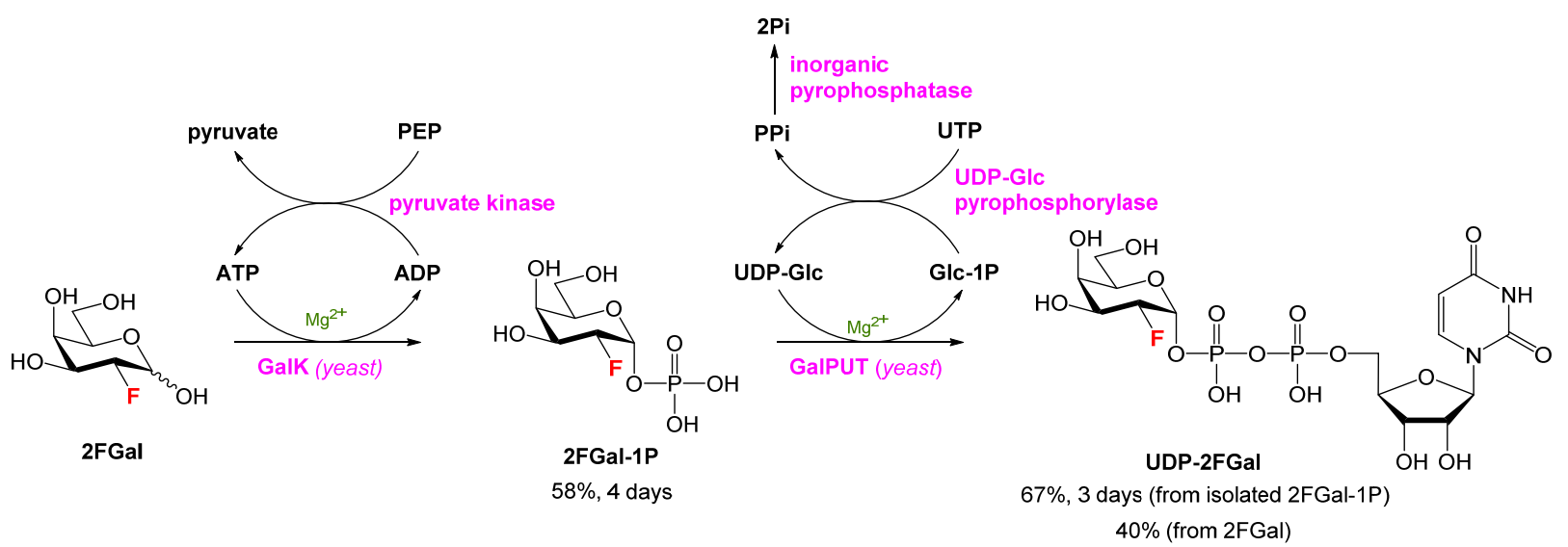

Scheme 2 Enzymatic synthesis of UDP-2FGal using a UDP-Glc regeneration cycle. ${ }^{21}$ 
Enzymatic synthesis of fluorinated nucleotide donors was also demonstrated by Barlow and Blanchard ${ }^{28}$. In this work, the authors describe the synthesis of both UDP-2FGal and UDP-3FGal from 2FGal and 3FGal respectively in preparative scale at near quantitative yields (based upon UDP-Glc conversion). This study also employs an alternative strategy to overcome problems associated with the purification of the desired UDP-sugar from the UDP-Glc generated in the catalytic cycle (Scheme 3). By using phosphoglucomutase (PGM) in place of UDP-Glc pyrophosphorylase the reaction was driven toward UDP-2FGal. The UDP-Glc cofactor could be removed from the system via further enzymatic breakdown, using glucose-6-phosphate dehydrogenase. This system does however require near stoichiometric quantities of costly UDP-Glc.

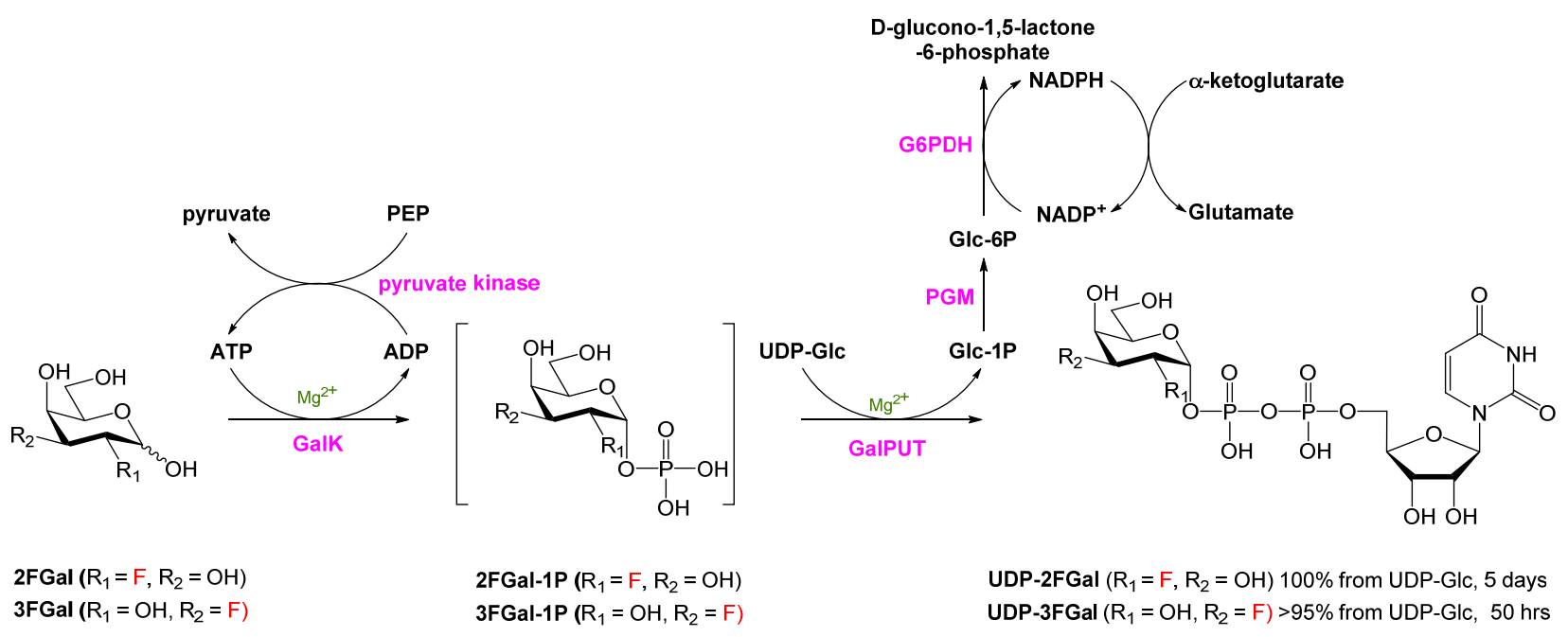

Scheme 3 Enzymatic synthesis of UDP-2FGal and UDP-3FGal with enzymatic breakdown of UDP-Glc. ${ }^{28}$

The utility and substrate scope of the GalK/GalPUT two-enzyme system was further expanded by Errey et al. ${ }^{22}$ In this work, the authors describe that UDP-6FGal can be synthesised from 6FGal using the GalK/GalPUT system (Scheme 4). Under identical reaction conditions, yields of UDP-2FGal were comparable to those achieved for the synthesis of UDP-Gal. In both cases near quantitative conversion was observed. In comparison, UDP-3FGal (64\%) and UDP-6FGal (37\%) were isolated in lower yields than the UDP-2FGal and the nonfluorinated product. Higher enzyme concentrations and longer reaction times were required for the synthesis of both UDP-3FGal and UDP-6FGal than for UDP-Gal and UDP-2FGal. It was unclear to the authors from their one-pot experiments which enzyme was rate limiting, but further experiments using chemically synthesised or commercially available sugar-1-phosphates of non-galactose sugars indicated that GalPUT has a relaxed substrate specificity and is able to turn over substrates not accepted by the two-enzyme system. This suggests GalK to be the rate limiting enzyme in the two-enzyme system. Indeed, these observations correlate with the 
early kinetic findings presented by Bessell et al., ${ }^{29}$ who found GalK to have a greater affinity for $2 \mathrm{FGal}$ than 3FGal and 6FGal. In addition to using the UDP-Glc regeneration cycle to aid product purification, Errey and coworkers $^{22}$ made use of GalK and GalPUT immobilised onto resin surfaces which offered the additional benefits of allowing facile enzyme recovery and simplified purification methods. This approach also meant the enzymes could be used in excess, allowing even reactions with low efficiency compared to natural substrates to be successfully achieved on a preparative scale.

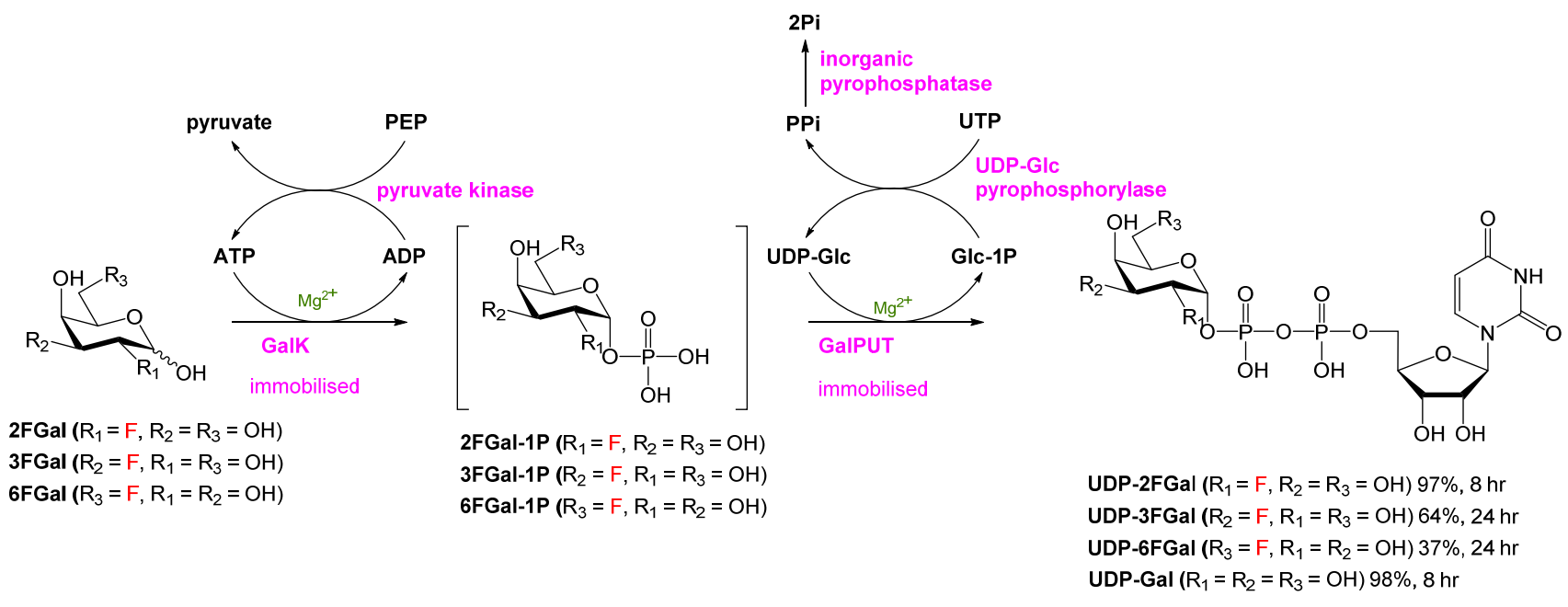

Scheme 4 Enzymatic synthesis of UDP-2FGal UDP-3FGal and UDP-6FGal using immobilised enzymes and the UDP-Glc regeneration cycle. ${ }^{22}$

The synthesis of UDP-4FGal from 4FGal was reported in the 2012 patent application of Nishimura et al. in two steps using E. coli derived GalK (EcGalK) and GalPUT (Scheme 5). ${ }^{30}$ The authors noted that the conversion for the second step (i.e. the formation of UDP-4FGal from 4FGal-1P) was 30\% lower if using GalPUT from yeast instead of E. coli was used, which highlights the value in considering enzymes from different sources.

Likewise, although initial kinetic studies using yeast GalK indicated a four-fold increase in $\mathrm{K}_{\mathrm{m}}$ for $4 \mathrm{FGal}$ relative to $\mathrm{Gal}$ and a $\mathrm{V}_{\max }$ of $10 \%$ relative to $\mathrm{Ga}^{29}$, the authors reported a surprisingly short reaction time $(1 \mathrm{~h})$ using E. coli GalK.

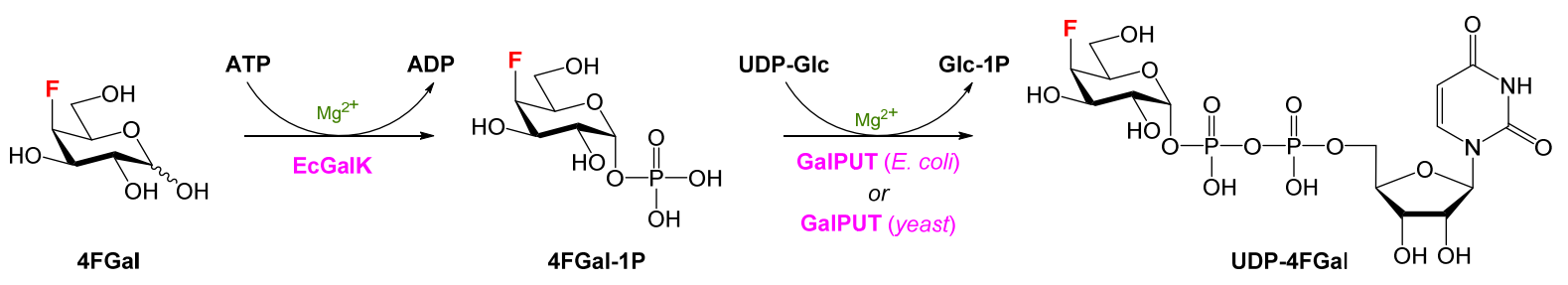

Scheme 5 Enzymatic synthesis of UDP-4FGal. ${ }^{30}$ 
6,6-Difluoro-galactose has been shown to be a substrate for GalK from Lactococcus lactis, a GalK demonstrated to have good substrate promiscuity against a large panel of sugars, with modifications at the 2, 3, 4 or 6 position (Scheme 6). ${ }^{31}$

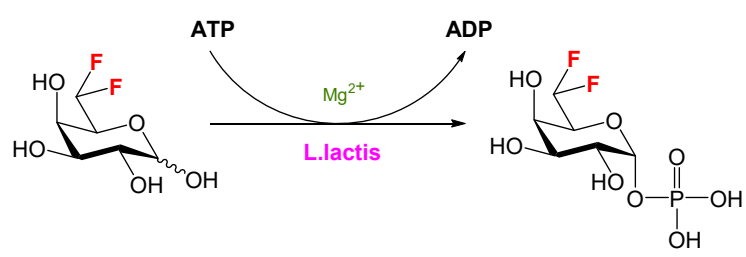

Scheme 6 Enzymatic phosphorylation of 6,6-difluoro-galactose. ${ }^{31}$

A series of furanosyl sugar nucleotides have been synthesised using a bacterial $\alpha$-D-glucopyranosyl-1-phosphate thymidylyltransferase, Cps2L from Streptococcus pneumoniae. ${ }^{32}$ In these reactions, chemically synthesised 6fluoro-galactofuranosyl-1-phosphate (anomeric mixture $\alpha / \beta=1.7 / 1$ ) was successfully converted into deoxythymidine diphosphogalactofuranose (dTDP-Galf) in the presence of Cps2L, deoxythymidine 5'triphosphate, $\mathrm{Mg}^{2+}$ and inorganic pyrophosphatase (Scheme 7). The conversion was moderate, only $19 \%$ in a 24 hour reaction. Later, the authors engineered $\mathrm{Cps} 2 \mathrm{~L}$ to expand its repertoire of nucleotide substrates including uridine and guanidine. ${ }^{33}$ One of mutants, Cps2L Q24S showed an enhanced activity in the conversion of 6fluoro-galactofuranosyl-1-phosphate $(\alpha / \beta=1.7 / 1)$ to dTDP-Galf with a conversion rate of c.a. $30 \%$ in 18 hours although its intended activity of uridylyltransfer was not detected with 6FGalf (Scheme 7).

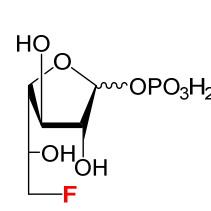

6FGalf-1-P

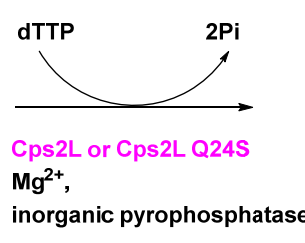

inorganic pyrophosphatas

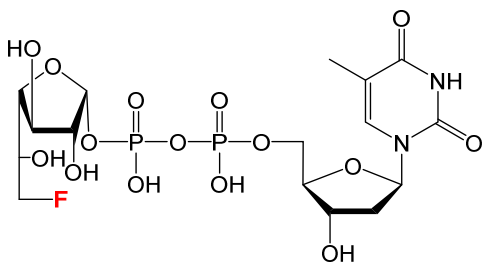

dTDP-6FGalf (19\%, 24 hours; 30\%, 18 hours)

Scheme 7 Enzymatic synthesis of dTDP-6FGalf. ${ }^{32-33}$ 


\subsubsection{Galactosyltransferases}

An understanding of the likely catalytic mechanism for galactosyltransferase enzymes along with metabolic studies using $2 \mathrm{FGal}$ (which causes inhibition of glycosylation), ${ }^{34}$ lead Hayashi et al. ${ }^{21}$ to propose that a strongly electron withdrawing fluorine atom at C-2 would result in enzyme inhibition. They report the $\mathrm{K}_{\mathrm{i}}(149 \pm 18 \mu \mathrm{M})$ versus $\mathrm{Km}(127 \pm 9 \mu \mathrm{M})$ for UDP-Gal indicating that the affinity of UDP-2FGal for the enzyme is comparable to the natural substrate and demonstrate UDP-2FGal to be a competitive inhibitor of $\beta-(1 \rightarrow 4)$ galactosyltransferase (GalT), with respect to UDP-Gal, fluorine at the 2-position unaffecting binding to the enzyme, but making the glycosidic bond inert to cleavage.

Whilst $\beta$-( $1 \rightarrow 4)$ GalT is inhibited by UDP-2FGal, it is capable of processing both the UDP-4FGal and UDP6FGal donors. To the best of our knowledge, there are no published examples UDP-3FGal being used as a donor in glycosyltransferase catalysed reactions.

4FGal and its applications, including the synthesis of UDP-4FGal detailed earlier, are reported in the previously mentioned 2012 patent application ${ }^{30}$ in which the use of UDP-4FGal as a donor for human derived $\beta-(1 \rightarrow 4)$

GalT is described. Incubation of the enzyme with a variety of $\beta$-glycosides of GlcNAc, followed by mass spectral characterisation indicated the formation of a peak corresponding to the desired disaccharides. However, the regio- and stereoselectivity was not unambiguously confirmed nor reaction conditions optimised (Scheme 8).
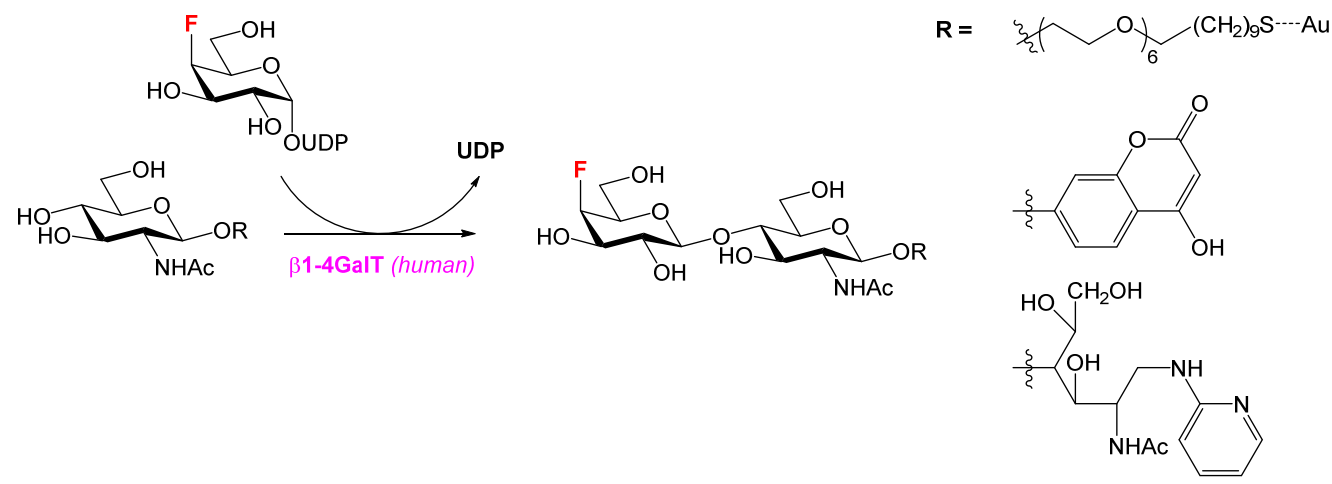

Scheme $8 \beta-(1 \rightarrow 4)$ GalT enzymatic synthesis of disaccharides containing $4 \mathrm{FGal} .{ }^{30}$ 
UDP-6FGal (obtained by chemical methods) was shown by Kodama et al. to be a substrate for $\beta$-( $1 \rightarrow 4)$ GalT from bovine milk, albeit at rates significantly lower than of UDP-Gal (Scheme 9). ${ }^{35}$ Nonetheless, increasing the enzyme concentration and reaction time allowed the enzyme to transfer UDP-6FGal to the natural GlcNAc acceptor to produce the fluorinated LacNAc analogue in 59\% yield, with the regio- and stereoselectivity confirmed by proton NMR spectroscopy. In an independent study ${ }^{36}$ using the same enzyme and acceptor, the authors were unable to detect transfer of UDP-6FGal to GlcNAc and demonstrated its ability as a competitive inhibitor of the enzyme. However, they noted that enzyme concentrations and reaction times were significantly reduced compared with the earlier study. These conflicting observations highlight the importance of screening multiple reaction conditions, and show that although reaction rates can be low compared to natural sugars, under optimised conditions use of enzymes can be a viable method of synthesis.

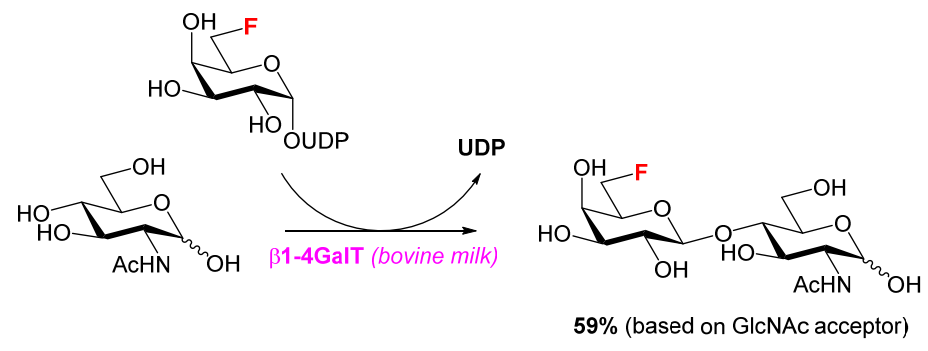

Scheme $9 \beta$ - $(1 \rightarrow 4)$ GalT enzymatic synthesis of disaccharides containing $6 \mathrm{FGal} .{ }^{35}$

\subsubsection{Phosphorylases}

Galactosyl 1-phosphate donors can be utilised by a phosphorylase from Bifidobacterium infantis

$($ BiGalHexNAcP) for the synthesis of $\beta-(1 \rightarrow 3)$ linked disaccharides. Furthermore, the phosphorylase enzyme along with a suitable kinase such as $E c G a l K$ can be used in a one-pot, two-enzyme system to afford the disaccharide directly from the reducing sugar. ${ }^{37}$ This system has been successfully employed with certain monofluorinated sugars.

Enzymatically synthesised 6FGal-1P was shown by Yan et al. to be substrate for BiGalHexNAcP and was able to be transferred to the 3-OH of a GalNAc acceptor with $\beta$-selectivity (Scheme 10). ${ }^{38}$ Preparative scale yields were high (ca. 80\%) and comparable to the natural donor Gal-1P. Furthermore, the enzyme was demonstrated to transfer 6FGal-1P to the unnatural fluorinated 6FGalNAc acceptor at yields comparable to GalNAc, yielding disaccharide fluorinated in both sugar units (see section 5). BiGalHexNAcP has optimum activity at pH 5.0 to 6.5, whereas EcGalK has optimum activity at $\mathrm{pH}>7.0$. When using natural donors and acceptors, $\mathrm{pH} 6.5$ was a 
good compromise giving reasonable overall yields, however for the fluorinated donors and acceptors, to maximise yields, a two-step process was used, at $\mathrm{pH} 7.5$ for the kinase reaction, and then once the reaction was complete as monitored by TLC, at $\mathrm{pH} 6.0$ with addition of BiGalHexNAcP.

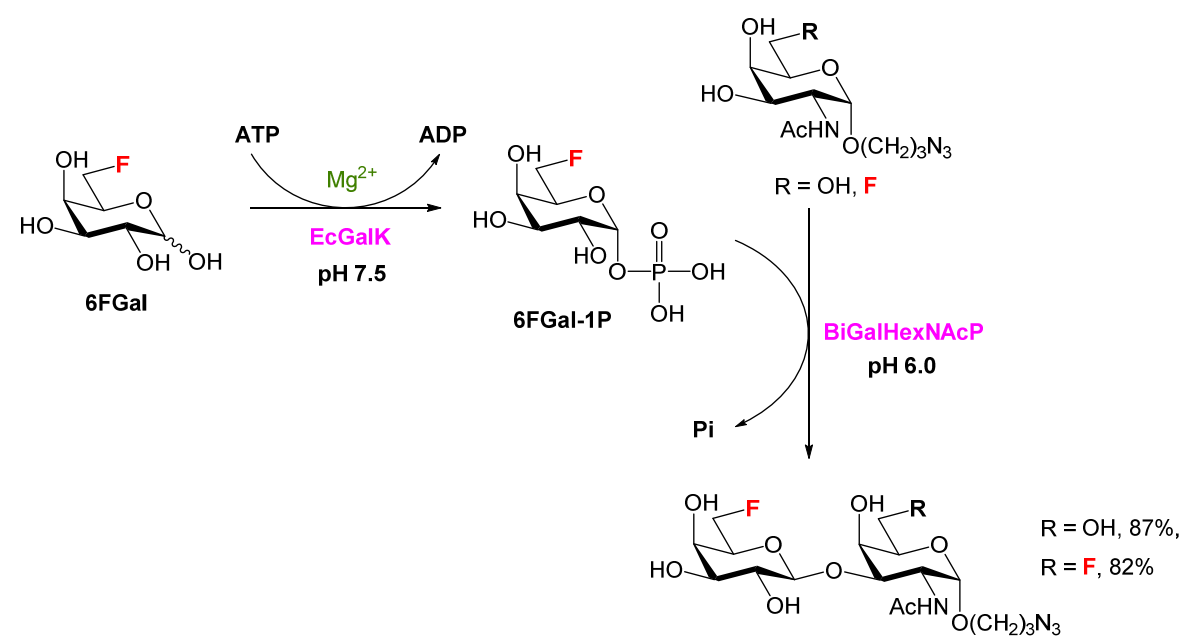

Scheme 10 One-pot two-step disaccharide synthesis using 6FGal. ${ }^{38}$

Whilst the authors initially reported that $2 \mathrm{FGal}-1 \mathrm{P}$ is not a substrate for the enzyme they later demonstrated that it can be transferred to either GalNAc or GlcNAc acceptors. ${ }^{39}$ As the Bifidobacterium infantis kinase BiGalK has relaxed substrate specificity and good activity at $\mathrm{pH} 6.5$ this removed the need for a two-step reaction (Scheme 11). Under the same reaction conditions, isolated yields (26-28\%) were much lower than for Gal-1P and 6FGal-1P however this activity and transformation is important, as 2-fluorinated UDP donors are inhibitors of glycosyltransferases as previously described, therefore the enzymatic routes to the synthesis of disaccharides containing 2-deoxy-2-fluoro sugar moiety at the reducing end are limited. Furthermore, it is worthwhile noting that in this two-enzyme reaction, the low conversion is due to the phosphorylase enzyme rather than the kinase, as $2 \mathrm{FGal}-1 \mathrm{P}$ can be synthesised by both $E c \mathrm{GalK}$ and $\mathrm{BiGalK}$ in yields greater than $90 \%$ under the conditions used in this system. 


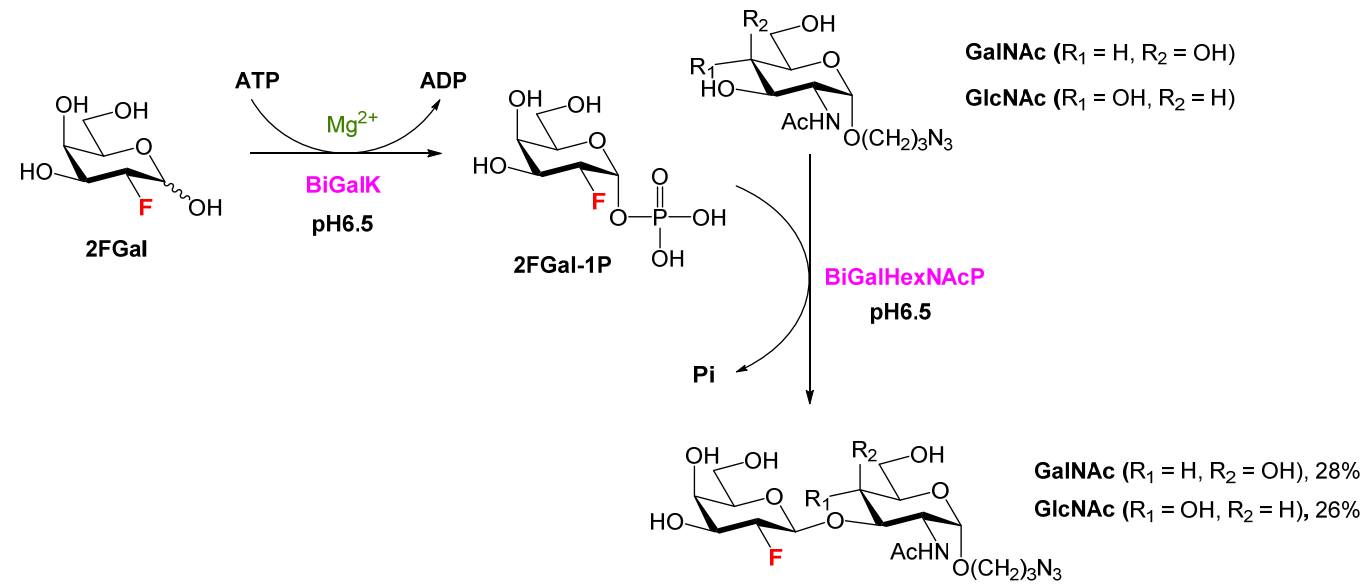

Scheme 11 One-pot disaccharide synthesis using $2 \mathrm{FGal} .{ }^{39}$

\subsubsection{Glycoside hydrolases}

As fluorinated carbohydrates have been reported to act as mechanism-based inhibitors of glycoside hydrolases particularly as a donor, ${ }^{6,12}$ it is not surprising to be able to find only few examples. O'Donohue et al. used pnitrophenyl $\beta$-D-galactofuranoside (pNP- $\beta \mathrm{Gal} f$ ) as a substrate of arabinofuranosidase from Thermobacillus xylanilyticus (AbfD3) to conduct transglycosidation reaction. ${ }^{40}$ To avoid extensive hydrolysis, the authors stopped the reaction in 30 minutes, and detected two transglycosidation product, $\beta-(1 \rightarrow 2)$ and $\beta-(1 \rightarrow 3)$ disaccharides, both of which were spectroscopically characterised (Scheme 12). Although the conversion rates or yields were not reported, the HPLC chromatogram showed that hydrolysis products were predominant. In another example, Ferrieres et al. conducted an enzymatic reaction with arabinofuranosidase (Araf51) from Clostridium thermocellum containing galactosyl furanose derivatives as substrates. ${ }^{41}$ The original intention was to use pNP- $\beta$ Galf as donor and octyl 6-fluoro- $\beta$-D-galactofuranoside (Oct 6FGalf) as acceptor, but the reaction yielded a mixture of various length oligosaccharides with both substrates as either donor or acceptor (Scheme 12). A few oligosaccharide products showed that Oct $6 \mathrm{FGalf}$ acted as donor albeit with the yield of only $1-6 \%$, which indicates that fluorination is not accepted well by the enzyme. 
<smiles>O=[N+]([O-])c1ccc(OC2OC3CC4COC3C(O)C4O2)cc1</smiles>

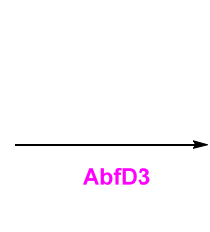

6FGalf-1-P

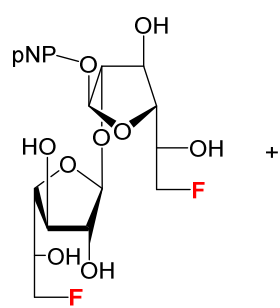

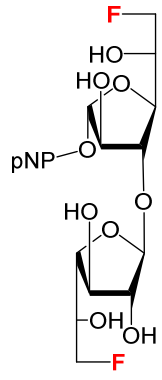

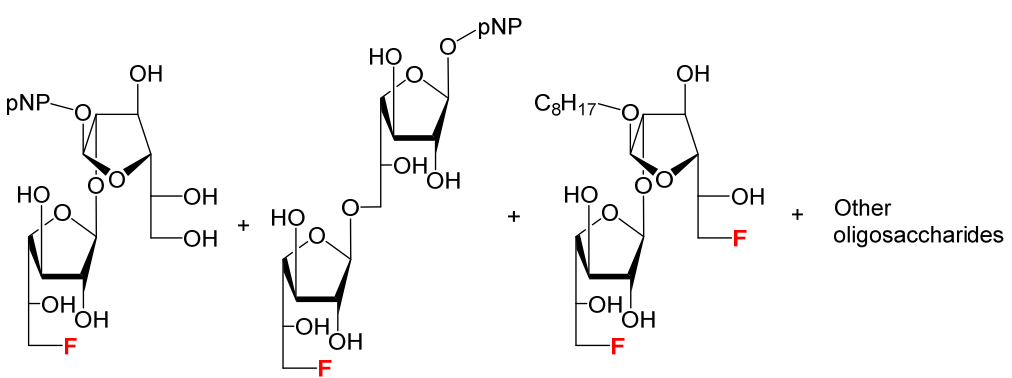

pNP-Galf<smiles>CCOC1(O)OC2CC(CO2)C1O</smiles>

Octyl 6FGalf

Scheme 12 Use of arabinofuranosidase to synthesise fluorinated di-galactofuranosides. ${ }^{40-41}$

\subsection{Fluorinated galactose acceptors}

Fluorinated galactose has also been used as an acceptor in enzymatic glycosylation, not as the monosaccharide but as a non-reducing galactose moiety as part of a disaccharide or larger oligosaccharide.

\subsubsection{Galactosyl- and $N$-acetylgalactosaminyltransferases}

The disaccharide Fuc- $\alpha-(1 \rightarrow 2)$ Gal is a motif common to both A and B blood group antigens. In biosynthetic pathways, $\alpha-(1 \rightarrow 3)$ galactosyltransferase $(\alpha-(1 \rightarrow 3) \mathrm{GalT})$ is responsible for transferring a Gal residue from UDP-Gal to this motif to give the B group antigen whereas $\alpha-(1 \rightarrow 3) \mathrm{N}$-acetylgalactosaminyltransferase $(\alpha-$ $(1 \rightarrow 3)$ GalNAcT) transfers a GalNAc residue to give the A antigen. ${ }^{42}$ Following chemical synthesis of the fluorinated acceptor analogue Fuc- $\alpha-(1 \rightarrow 2) 6 \mathrm{FGal}-\beta-\mathrm{O}\left(\mathrm{CH}_{2}\right)_{7} \mathrm{CH}_{3}$, biosynthesis of the modified antigens was achieved using both natural donors (Scheme 13), ${ }^{43}$ however the enzymes $\alpha-(1 \rightarrow 3)$ GalT and $\alpha-(1 \rightarrow 3)$ GalNAcT had a lower affinity for the fluorinated acceptor than the natural acceptor $(\alpha-(1 \rightarrow 3)$ GalT $30 \%$ activity, $\alpha$ $(1 \rightarrow 3)$ GalNAcT $43 \%$ activity relative to the native disaccharide). Fuc- $\alpha-(1 \rightarrow 2) 4$ FGal was not a substrate (or inhibitor) for either enzyme, demonstrating the importance of the 4-position hydroxyl for these enzymatic transformations. Fuc- $\alpha-(1 \rightarrow 2)-3$ FGal was shown to be a weak competitive inhibitor of both enzymes. 


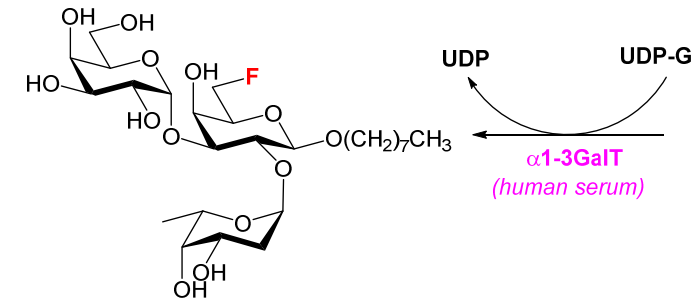

Blood group antigen $B$ trisaccharide

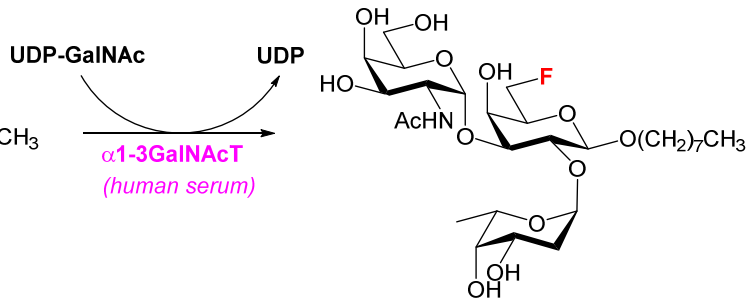

Blood group antigen $A$ trisaccharide

Scheme 13 6FGal as acceptor in enzymatic synthesis using galactosyl and $N$-acetylgalactosamine transferases. ${ }^{43}$

\subsubsection{Sialyltransferases and trans-sialidases}

Fluorinated sugars have been utilised as acceptor substrates for sialylation using both sialyltransferases (SiaT) and trans-sialidases. The enzymatic incorporation of fluorinated sialic acid derivatives as donors is discussed in section 9.

The disaccharide 2FGal $\beta-(1 \rightarrow 3)$ GalNAc is a suitable acceptor substrate for the $\alpha-(2 \rightarrow 3)$ Pasteurella multocida SiaT 1 (PmST1). The trisaccharide Neu5Ac $\alpha-(2 \rightarrow 3) 2 \mathrm{FGal} \beta-(1 \rightarrow 3)$ GalNAc $\alpha-\mathrm{O}-\left(\mathrm{CH}_{2}\right)_{3} \mathrm{~N}_{3}$ was synthesised in preparative scale in excellent yields from the corresponding disaccharide acceptor $2 \mathrm{FGal} \beta$ $(1 \rightarrow 3)$ GalNAc $\alpha-\mathrm{O}-\left(\mathrm{CH}_{2}\right)_{3} \mathrm{~N}_{3}$ and Neu5Ac using this enzyme, coupled with the CMP sialic acid synthetase from Neisseria meningitidis (NmCSS) in a one-pot, two-enzyme system (Scheme 14). Yields were comparable to the natural acceptor Gal $\beta-(1 \rightarrow 3)$ GalNAc under the same reaction conditions. ${ }^{39}$

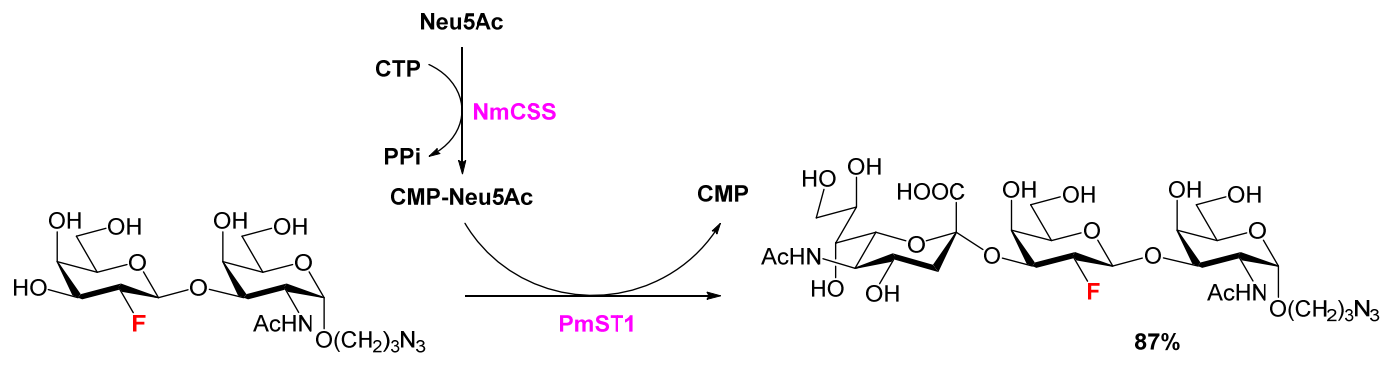

Scheme 14 2FGal as acceptor in enzymatic synthesis using SiaT PmST1. ${ }^{39}$

Incubation of dodecyl 2FGal, dodecyl 4FGal and dodecyl 6FGal with mouse melanoma B16 cells showed toxicity with $6 \mathrm{FGal}$ and $4 \mathrm{FGal}$, however $2 \mathrm{FGal}$ produced a glycosylated product, corresponding to a sialylated product by mass spectrometric analysis. ${ }^{44}$ This observation was confirmed through use of a $\alpha-(2 \rightarrow 3)$ sialidase from S. typhimurium (cloned in E. coli) to be a fluorinated analogue of the ganglioside GM-4 (Neu5Ac $\alpha-(2 \rightarrow 3)$ 
Gal $\beta$ ceramide) (Scheme 15), indicating that cellular $\alpha-(2 \rightarrow 3)$-SiaT are able to utilise $2 \mathrm{FGal}$ as an acceptor for the synthesis.

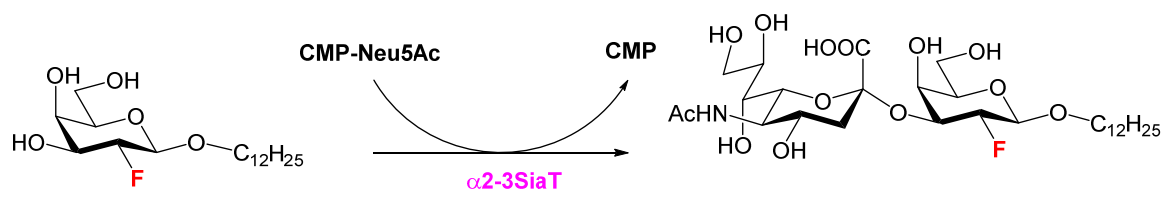

Scheme 15 FGal as acceptor for cellular $\alpha-(2 \rightarrow 3)$ SiaT from S. typhimurium. ${ }^{44}$

Disaccharides terminated with $2 \mathrm{FGal}$ have also been shown to be suitable acceptors for the T. cruzi transsialidase (TcTs) mediated transfer of Neu5Ac from the heavily sialylated mammalian protein fetuin. A crude cell lysate can be used for the transformation, and using TcTs with fetuin as the Neu5Ac donor removes the requirement for CMP-Neu5Ac and the enzymes associated with its synthesis. Using this system, Allman et al. ${ }^{45}$ demonstrated the synthesis of the trisaccharides Neu5Ac $\alpha-(2 \rightarrow 3)$ FGal $\beta-(1 \rightarrow 4)$ GlcNAc and Neu5Ac $\alpha-$ $(2 \rightarrow 3)$ 2FGal $\beta-(1 \rightarrow 3)$ GlcNAc, from the corresponding chemically synthesised disaccharides, with the regioand stereo-selectivity of the enzyme confirmed via NMR spectroscopy (Scheme 16). For both $\beta-(1 \rightarrow 3)$ and $\beta$ $(1 \rightarrow 4)$ linked disaccharides, yields for the sialylation were less than obtained for the natural disaccharides, although in the case of the natural $\beta-(1 \rightarrow 4)$ disaccharide, the use of an OBn anomeric protecting group instead of OTBDMS at the reducing end could also affect the affinity of the enzyme for the acceptor.
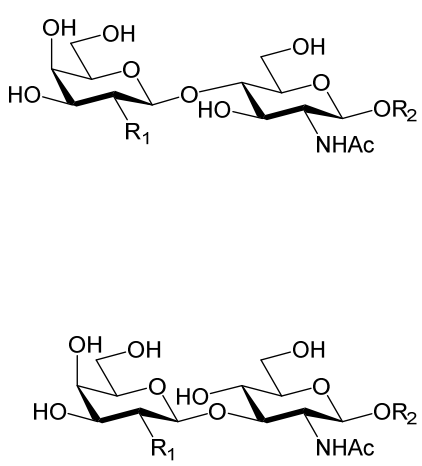
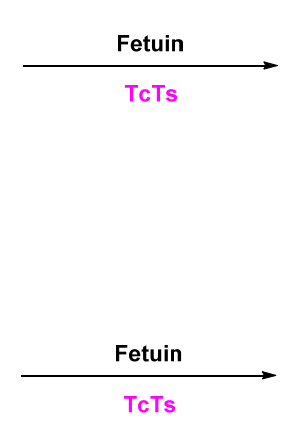

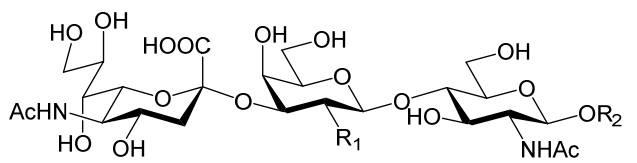

$\mathrm{R}_{1}=\mathrm{OH}, \mathrm{R}_{2}=\mathrm{Bn} 83 \%$

$R_{1}=F, R_{2}=$ TBDMS $45 \%$

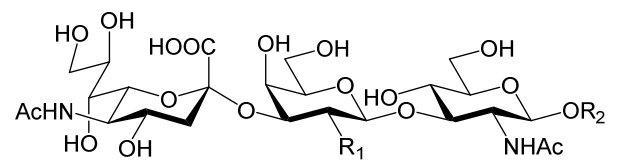

$\mathrm{R}_{1}=\mathrm{OH}, \mathrm{R}_{2}=\mathrm{Bn} 63 \%$ $\mathrm{R}_{1}=\mathrm{F}, \mathrm{R} 2=\mathrm{TBDMS} 30 \%$

Scheme 16 2FGal as acceptor in enzymatic synthesis using TcTs. ${ }^{45}$

4FGal $\beta$ - $(1 \rightarrow 4)$ GlcNAc $\beta-(1 \rightarrow 2)$ Man was shown to be a substrate for both human and recombinant rat liver $\alpha-(2 \rightarrow 6)$ SiaT with a good sialylation rate relative to the natural trisaccharide Gal $\beta-(1 \rightarrow 4)$ GlcNAc $\beta$ - $(1 \rightarrow 2)$ 
Man, and also a poor substrate for recombinant rat liver $\alpha-(2 \rightarrow 3) \operatorname{SiaT}^{46}$ 3FGal $\beta-(1 \rightarrow 4)$ GlcNAc $\beta-(1 \rightarrow 2)$ Man showed low-level sialylation with both the human and rat liver $\alpha-(2 \rightarrow 6)$ SiaT in screening reactions (Scheme 17). In scale-up synthesis, the fluorinated tetrasaccharides Neu5Ac $\alpha-(2 \rightarrow 3) 4 \mathrm{FGal} \beta-(1 \rightarrow 4)$ GlcNAc $\beta-(1 \rightarrow 2)$ Man and Neu5Ac $\alpha-(2 \rightarrow 6) 4$ FGal $\beta-(1 \rightarrow 4)$ GlcNAc $\beta-(1 \rightarrow 2)$ Man were able to be synthesised in low milligram quantities from 4FGal $\beta-(1 \rightarrow 4)$ GlcNAc $\beta-(1 \rightarrow 2)$ Man using rat liver recombinant $\alpha 2-3$ SiaT or rat liver $\alpha 2-6$ SiaT respectively. Reactions were performed over 7 days with CMP-Neu5Ac added in batches every 2 days, along with calf intestinal alkaline phosphatase to break down the released CMP, which otherwise inhibits the sialyl transferase. ${ }^{47}$

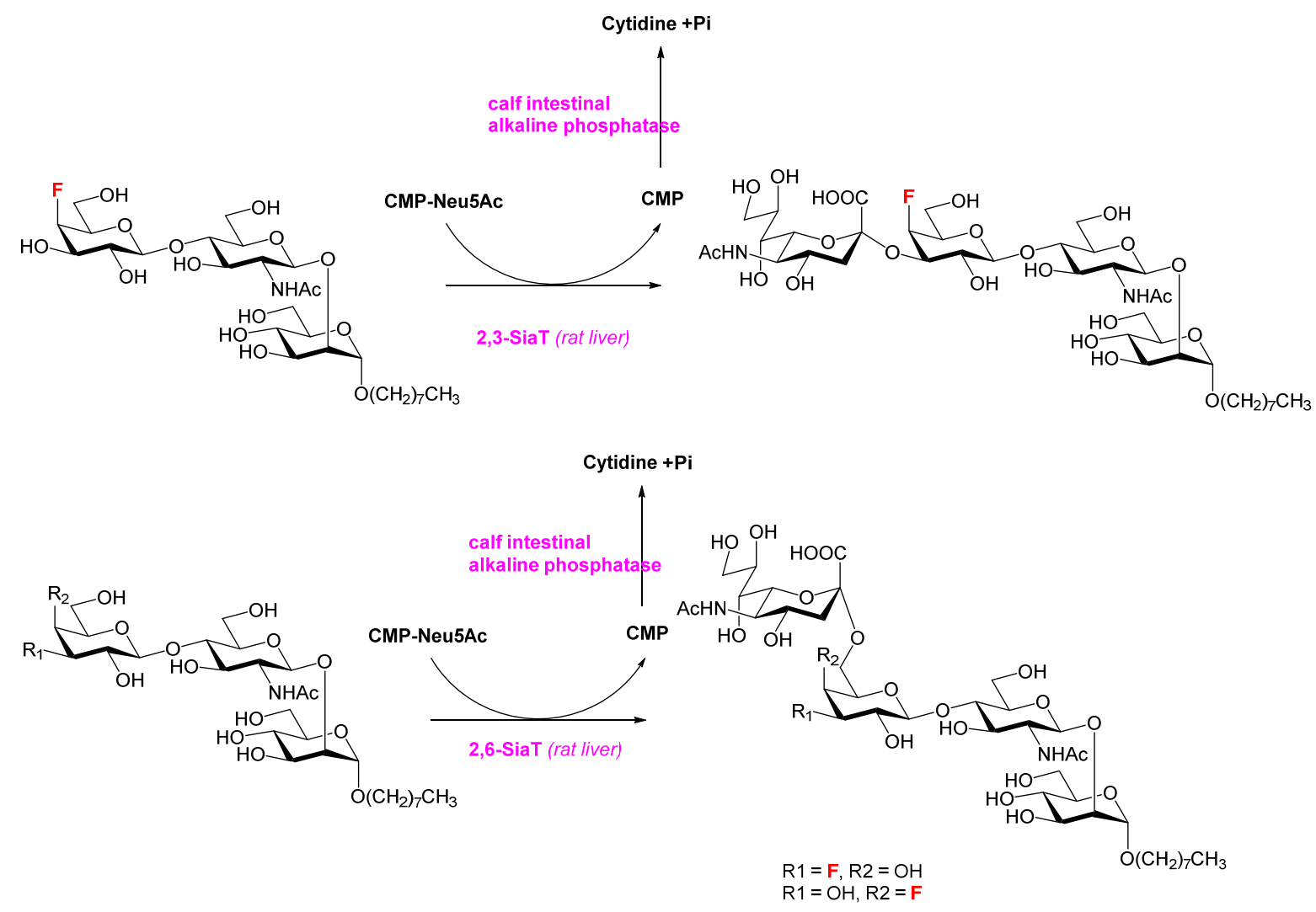

Scheme 17 Trisaccharides containing 3FGal or 4FGal as acceptors in enzymatic synthesis using $\alpha-(2 \rightarrow 3)$ and $\alpha-(2 \rightarrow 6)$ SiaTs. $^{46}$

Rat liver $\alpha-(2 \rightarrow 3)$ - and $\alpha-(2 \rightarrow 6)$ - SiaTs also tolerate 4-deoxy-4-fluorogalactoside acceptors with further modifications at the reducing end. The Neu5Ac $\alpha-(2 \rightarrow 3) 4 \mathrm{FGal}$ and Neu5Ac $\alpha-(2 \rightarrow 6) 4 \mathrm{FGal}$ glycosides depicted in Scheme 18 were detected via HPLC and mass spectrometry following reaction with the appropriate enzyme and CMP-Neu5Ac, however the connectivity was not unambiguously determined. In all examples, kinetic data revealed the reaction to proceed slower for the fluorinated donor than for the unmodified. ${ }^{30}$ 


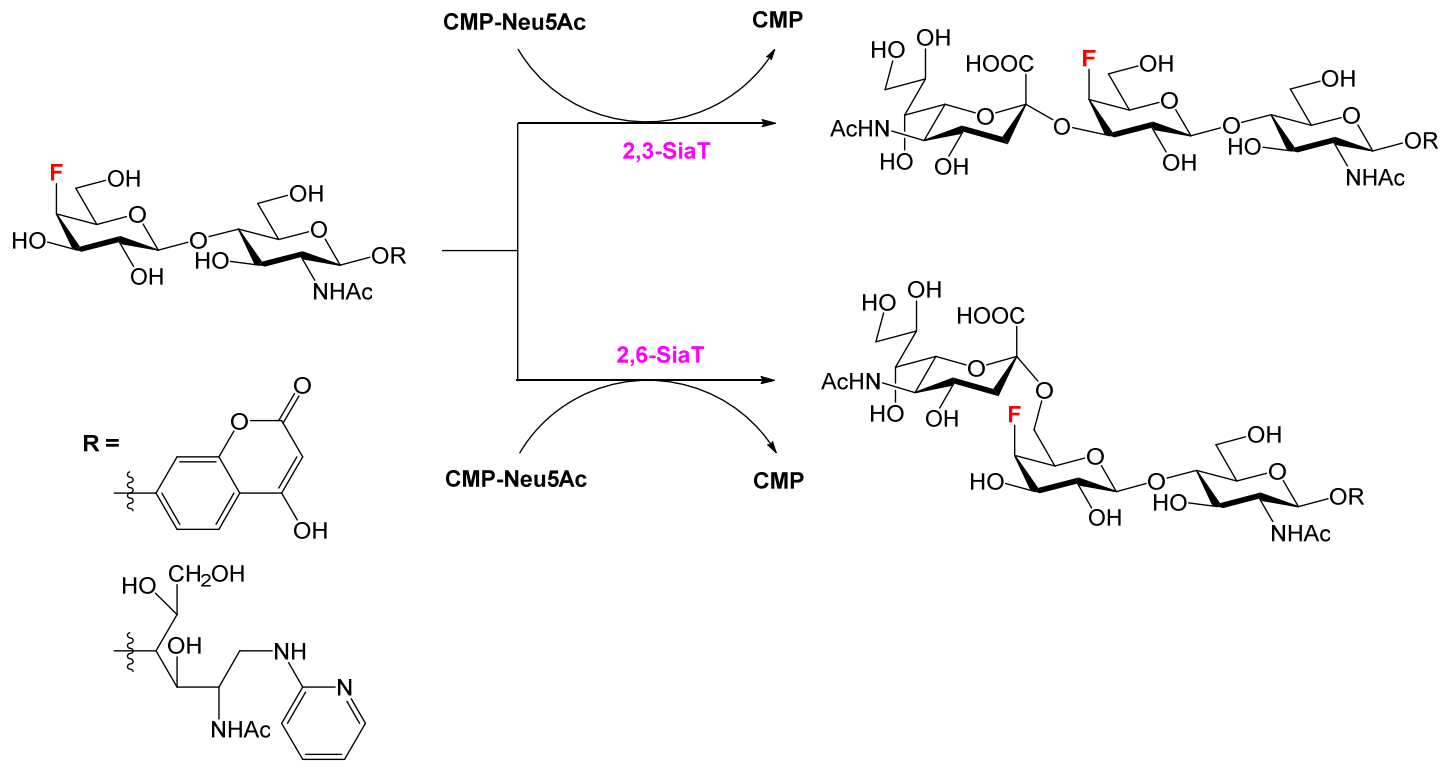

Scheme 18 Disaccharide containing 4FGal as acceptor in enzymatic synthesis using $\alpha-(2 \rightarrow 3)$ and $\alpha-(2 \rightarrow 6)$ SiaTs. ${ }^{30}$

Completing the fluorinated galactose sugars, disaccharides containing 6FGal were shown to be excellent acceptors for the $\alpha-(2 \rightarrow 3)$ SiaT PmST1 (Scheme 19). Combined with NmCSS in the one-pot, two-enzyme system introduced above, the $\alpha-(2 \rightarrow 3)$ linked sialosides have been synthesised in milligram quantities, in yields comparable to the natural acceptor under the same reaction conditions. Stereo- and regioselectivity of the sialylation were confirmed by spectroscopic methods. ${ }^{38}$ 


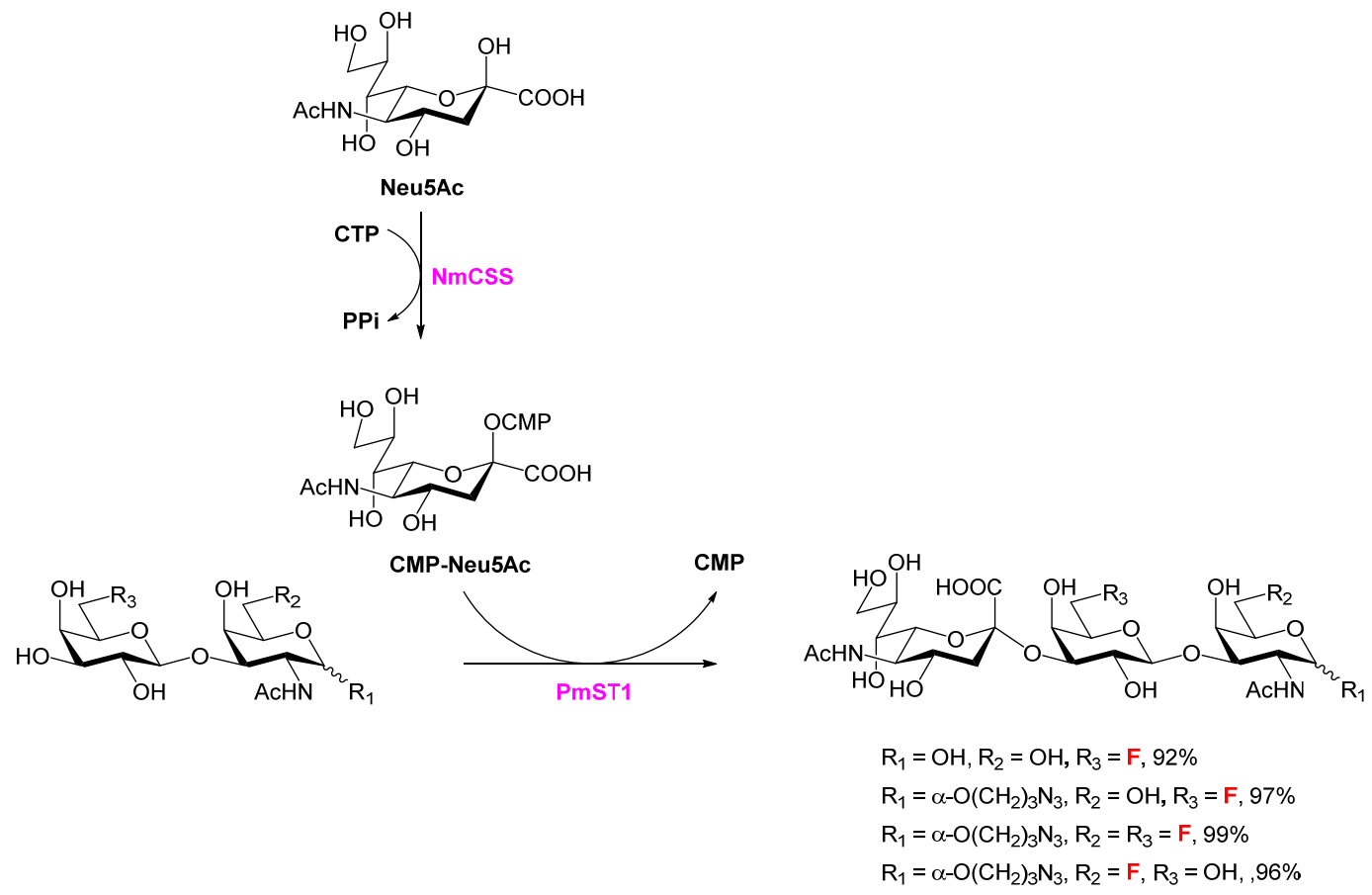

Scheme 19 3FGal and 4FGal containing trisaccharides as acceptors in enzymatic synthesis using $\alpha-(2 \rightarrow 3)$ and $\alpha-(2 \rightarrow 6)$ SiaTs. $^{38}$

\section{Fluorinated Glucose}

The synthetic strategy toward deoxyfluoro- $\alpha$-D-glucopyranosyl phosphates and UDP-deoxyfluoro- $\alpha$-Dglucopyranoside donors differs from that described above for their galactosyl analogues. Although GalK tolerates the introduction of fluorine, it has a high stringency toward galactose stereochemistry and Westwood demonstrated many years ago that 4FGlc was neither a substrate nor an inhibitor of yeast GalK. ${ }^{26}$ Even a mutant GalK, which is known to possess relatively relaxed substrate specificity at the C-4 position was unable to efficiently phosphorylate 3FGlc, 4FGlc or 6FGlc. ${ }^{48}$ Indeed, to the best of our knowledge, no kinases have been reported in the literature that are capable of efficiently processing deoxyfluorinated glucopyranosides. With the lack of suitable kinases, other enzymes have been evaluated in order to enzymatically access deoxyfluoro- $\alpha$-D-glucopyranosyl phosphates. In particular, the hexokinase/phosphoglucomutase approach has shown promise. Early kinetic studies revealed 2FGlc and 2,2FFGlc are good substrates for yeast hexokinase with affinities similar to Glc. ${ }^{29}$ A hexokinase/phosphoglucomutase approach, followed by conversion with UDP-glucose-1-pyrophosphorylase, has been used in the synthesis of UDP-2FGlc (Scheme 20). ${ }^{11}$ The UDPglucose-1-pyrophosphorylase mediated step was investigated further, and it was found that the activity of this enzyme decreases by a factor of 30 for the 2FGlc-1P compared with Glc-1P, and a six-day reaction time was 
required to achieve $60 \%$ conversion of the $2 \mathrm{FGlc}$. Following purification, UDP-2FGlc could be isolated in milligram quantities in $38 \%$ yield. The authors then attempted to extend this methodology to the synthesis of ${ }^{18} \mathrm{~F}$ radio-labelled UDP-2FGlc. However, limited radiochemical yields led them to employ a chemo-enzymatic protocol whereby the 2FGlc-1P was first synthesised chemically. Enzymatic conversion to UDP-2FGlc was carried out in the void volume of an anion exchange cartridge, and using increased concentrations of UDPglucose-1-pyrophosphorylase, the product was isolated with a radiochemical yield of $20 \%$ with a total synthesis time of 110 minutes from 2FGlc.

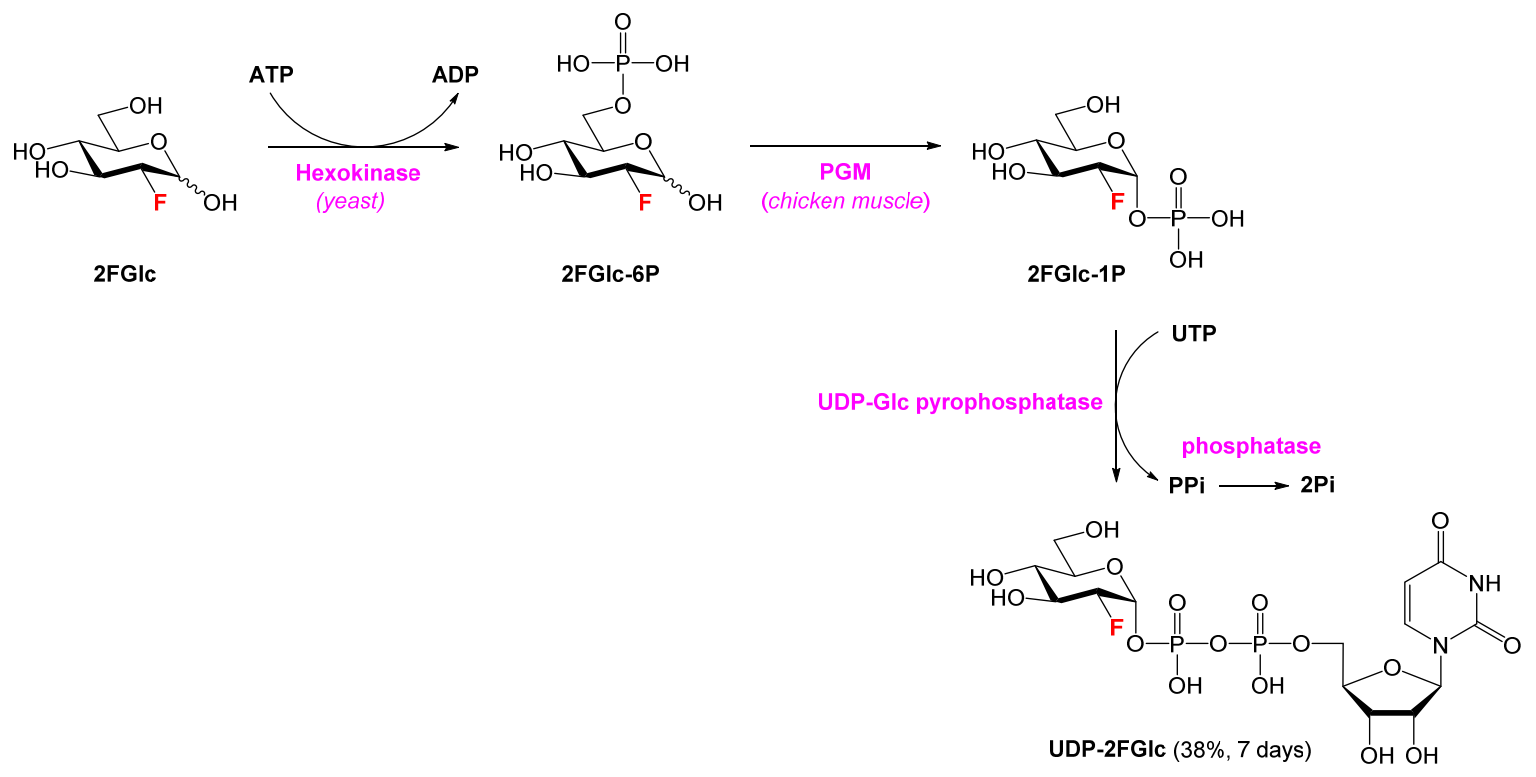

Scheme 20 Enzymatic synthesis of UDF-2FGlc using hexokinase, phosphoglucomutase and UDP-Glc pyrophosphorylase. ${ }^{11}$

On the other hand, 3FGlc and 4FGlc are poor substrates for yeast hexokinase. ${ }^{29}$ Indeed, the hexokinase/PGM approach to synthesising 3FGlc-1P and 4FGlc-1P from 3FGlc and 4FGlc respectively was evaluated by Caputi et al. who found only a low conversion to the anomeric phosphates, and abandoned this pathway in favour of other more synthetically viable routes. Namely, they successfully demonstrated the combination of UDP-Gal synthesis with uridine-5'-diphosphogalactose-4-epimerase (GalE) for the synthesis of fluorinated UDP-sugars with the glucose configuration. UDP-6FGal was enzymatically synthesised using the GalK/GalPUT system described earlier. ${ }^{22}$ Subsequent incubation with GalE resulted in inversion of the stereochemistry at C-4' to give UDP-6FGlc, with the equilibrium reached after $3 \mathrm{hrs}$ in favour of the glc-configured product (UDP-6FGlc:UDP- 
6FGal 3:1) (Scheme 21). ${ }^{48}$ The extension to the GalE approach to other members of the deoxyfluoro glucosyl pyranosides is yet to be explored, however the synthesis of UDP-4FGlc is obviously not possible using this methodology.

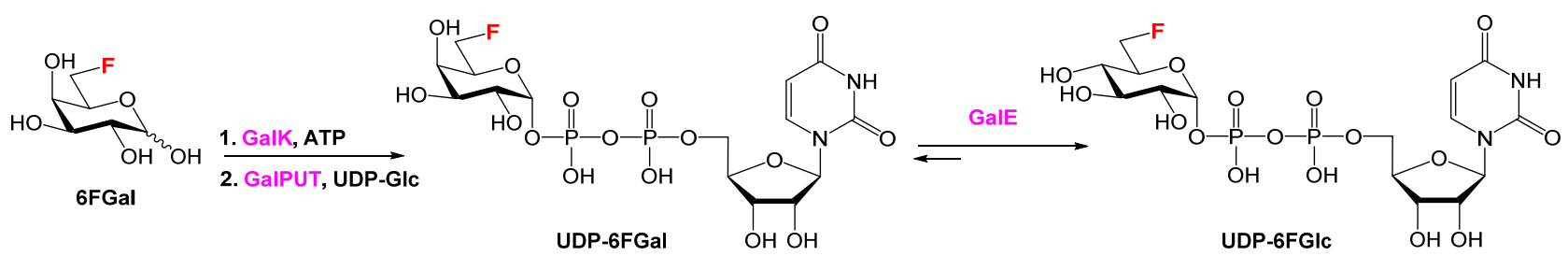

Scheme 21 Enzymatic synthesis of UDP-6FGlc using uridine-5'-diphosphogalactose-4-epimerase (GalE). ${ }^{48}$

The feasibility of UDP-6FGlc as a donor for glucosyltransferase mediated synthesis was demonstrated using the oat root transferase SAD10, whose physiological role is the generation of $O$-glucosyl $N$-methylanthranilate ester. Indeed, UDP-6FGlc (synthesised from UDP-6FGal by GalE as described earlier) ${ }^{48}$ was a substrate for the SAD10, which transferred the 6FGlc to $N$-methylanthranilic acid (MNA-H). The reaction did not proceed to completion, but instead an equilibrium was reached within 20 hours, which showed approximately $31 \%$ conversion of UDP-6FGlc into 6FGlc-NMA. This ratio was similar for the natural donor UDP-Glc. ${ }^{48}$ Certain glycosyltransferases from bacterial sources have been revealed to catalyse their traditional reaction in reverse, and this offers an alternative strategy toward the synthesis of NDP-sugars that does not require multiple enzymes (e.g. kinases, pyrophosphorylases, phosphatase) described above. However, careful selection of the activated aromatic glycoside donors is required to overcome thermodynamic limitations in order to drive the formation of NDP-sugar. Extensive screening of a range of aromatic glycosides and variants of oleandomycin glycosyltransferase (OleD) from Streptomyces antibioticus revealed a variant TDP16 and 2-chloro-4nitrophenyl- $\beta$-D-glucopyranoside to be the best enzyme/donor combination for the reaction to occur in reverse. Furthermore, little to no donor hydrolysis was observed under the conditions. The authors went on to show TDP16 was able to convert 2-chloro-4-nitrophenyl-6-fluoro- $\beta$-D-glucopyranoside to either UDP-6FGlc or TDP6FGlc when reacted in a 1:1 molar ratio with UDP or TDP, respectively (Scheme 22). For both NDPs, the conversion was greater than $90 \%$ after 21 h. ${ }^{49}$ 


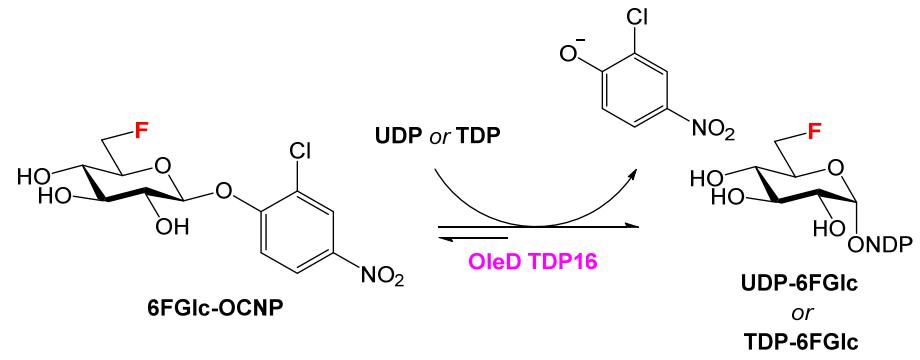

Scheme 22 Enzymatic synthesis of UDP- or TDP-6FGlc using the reverse reaction of a glycosyltransferase, TDP16, an engineered variant of OleD. ${ }^{49}$

Further to this, it was shown that TDP16 could be used in a one-pot glycosylation procedure whereby the NDPdonor produced by the reverse reaction could be coupled in situ to an aglycone acceptor by the same or an additional glycosyltransferase working in the forward direction. Thus, from 2-chloro-4-nitrophenol-6-fluoro- $\beta$ D-glucopyranoside, the 6F-glucosides of both 4-methylumbelliferone (MBU) and vancomycin were detected using either a single enzyme (MBU) or double enzyme (vancomycin) coupled system in the presence of a molar equivalent of UDP. After a reaction time of 24 hours, $70 \%$ and $40 \%$ conversion from the donor were observed for the MBU and vancomycin glycosides respectively. ${ }^{49}$

Cps2L (Streptococcus pneumoniae) is the enzyme that catalyses the synthesis of deoxythymidine diphosphate glucopyranose (dTDP-Glc), from glucose-1-phosphate. When evaluated against a panel of fluorinated glucose phosphates (2FGlc, 3FGlc, 4FGlc, 6FGlc, 4FGal, and 2,2F 2 Glc) and 2FMan, it was shown to exhibit broad substrate specificity as it could convert all the substrates almost as effectively as for the natural substrate Glc-1P within 20-30 minutes (Scheme 23) ${ }^{50}$ Kinetic analysis revealed 6FGlc behaved in a very similar manner to Glc, and this is consistent with previous studies that indicated that the 6'-hydroxyl group of Glc-1P made the smallest contribution to interactions with the enzyme ${ }^{51}$. Fluorination at the 3 ' position resulted in the most substantial decrease of $\mathrm{k}_{\mathrm{cat}} / \mathrm{K}_{\mathrm{m}}$ compared to Glc-1P and other monofluorinated substrates, and to a somewhat lower extent, the enzyme did not tolerate well a change in stereochemistry at the 4 position. The 2,2 difluorinated version of Glc-1P maintained binding affinity, but with a decreased turnover number. ${ }^{50}$ The same set of fluorinated sugars were evaluated with the enzyme guanosine diphosphate-mannopyranose pyrophosphorylase (GDP-ManPP) from Salmonella enterica (Scheme 23). ${ }^{50}$ When given long enough reaction times, the enzyme was capable of turning over all the sugars assayed. 2FGlc-1P, and 2F2FGlc-1P were converted almost quantitatively within $2.5 \mathrm{~h}$, which is consistent with the timeframe for the natural substrate Man-1P. 3FGlc-1P, 4FGlc-1P, 4FGal-1P and 6FGlc-1P required longer reaction times for similar rates of 
conversion. The authors suggested the possible use of the enzymes and products in the synthesis of unnatural glycans, however, further results have not been reported yet.
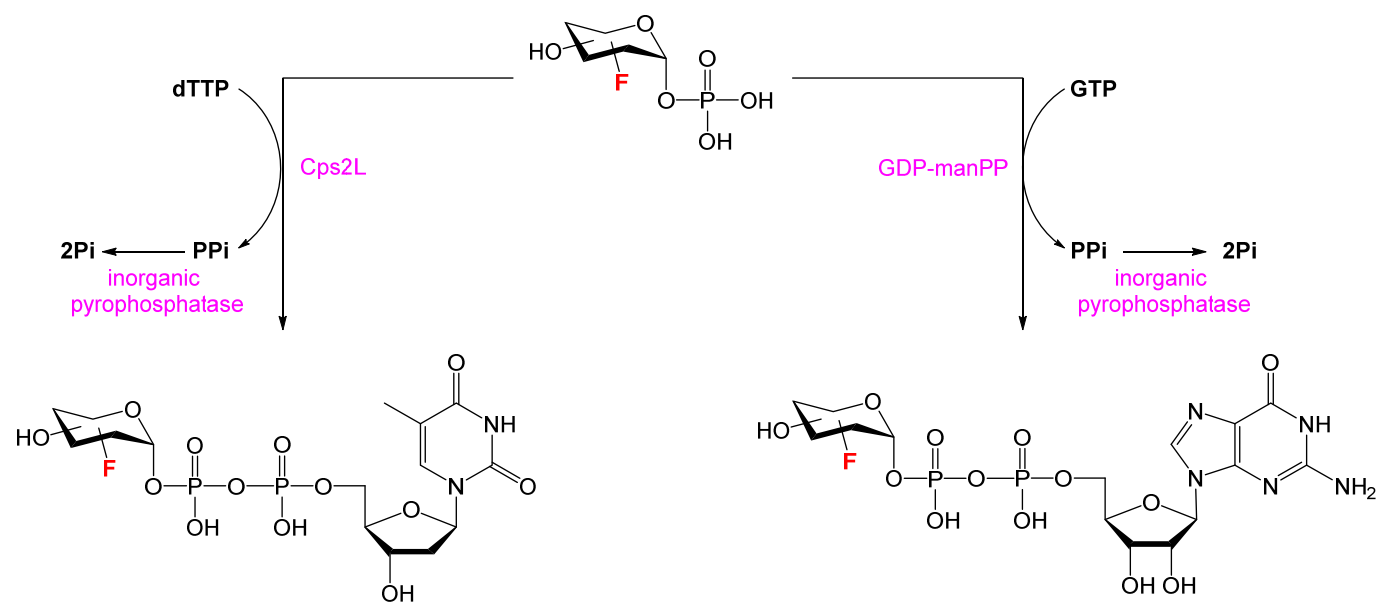

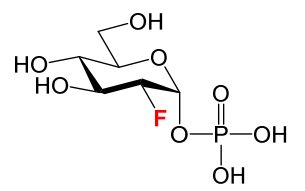

2FGIC-1P

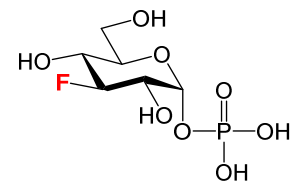

3FGIC-1P

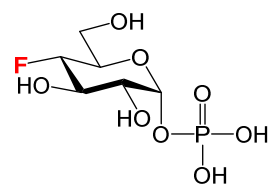

4FGIC-1P

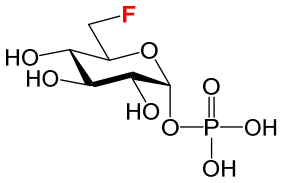

6FGIC-1P

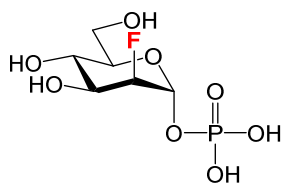

2FMan-1P

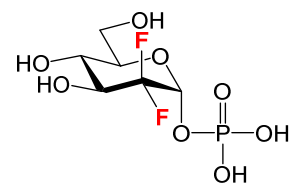

2F2FGIC-1P

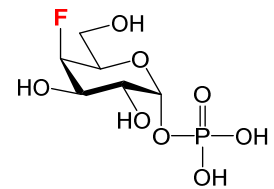

4FGal-1P

Cps2L, \% conversion

GDP-manPP, \% conversion

2FGlc-1P

3FGlc-1P

4FGIC-1P

6FGlc-1P

2FMan-1P

2F2FGlc-1P

4FGal-1P
94

93

94

95

93

95

93
95

87

79

94

98

93

78

Scheme 23 Enzymatic synthesis of a series of fluorinated TDP and GDP-sugars using Cps2L and GDPmannopyranose pyrophosphorylase. ${ }^{50}$ 
The use of fluorinated glucose as an acceptor of glycosyltransferases is rare. Notable examples are the synthesis of fluorinated trehalose analogues. Trehalose-6-phosphate (T6P) synthase (OtsA) from E. coli is a retaining glucosyltransferase that transfers glucose from UDP-glucose to the anomeric centre of glucose-6-phosphate (G6P) to synthesise a non-reducing disaccharide phosphate, trehalose-6-phosphate (Scheme 24). 2-Fluoro-, 3fluoro- and 4-fluoro-glucose-6-phosphates and 2-fluoro-mannose-6-phosphate were used as the acceptor for kinetic studies, which revealed all the fluorinated acceptors acted as a substrate albeit with lower catalytic efficiencies. ${ }^{52}$ Products were not analysed spectroscopically except for 2-fluoro-trehalose-6-phosphate, which was fully characterised. ${ }^{53}$ 2-F T6P then undergoes phosphate hydrolysis by T6P specific phosphatase, OtsB to yield 2-fluoro-trehalose (Scheme 24). Later, the Swarts group used a trehalose synthase (TreT) from Thermoproteus tenax to synthesise fluorinated trehalose analogues. ${ }^{54}$ This enzyme is a retaining glucosyltransferase that uses UDP-glucose as a donor and glucose as acceptor, and thus able to synthesise trehalose in one step unlike the OtsA approach. The authors demonstrated that TreT could accept 2-fluoro-, 3fluoro- and 6-fluoro-glucose as acceptor substrates while it did not react with 4-fluoro-glucose (Scheme 24). In contrast to OtsA, TreT showed comparable activities with fluorinated glucose acceptors to that of glucose, and particularly, the highest activity was measured with 2-fluoro-glucose. 2-Fluoro-, 3-fluoro- and 6-fluorotrehalose analogues were fully characterised.

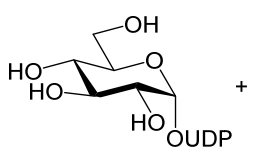

UDP-GIC

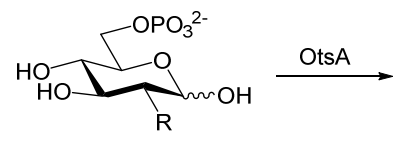

Glc-6P or 2FGlc-6P $\mathrm{R}=\mathrm{OH}$ or $\mathrm{F}$

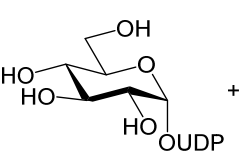

UDP-Glc

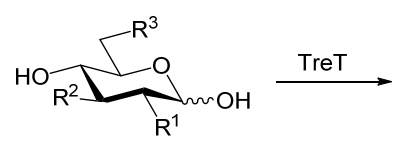

Fluorinated glucose

2FGlc: $R^{1}=F, R^{2}, R^{3}=\mathrm{OH}$

3FGlc: $R^{2}=F, R^{1}, R^{3}=O H$

6FGlc: $R^{3}=F, R^{1}, R^{2}=O H$
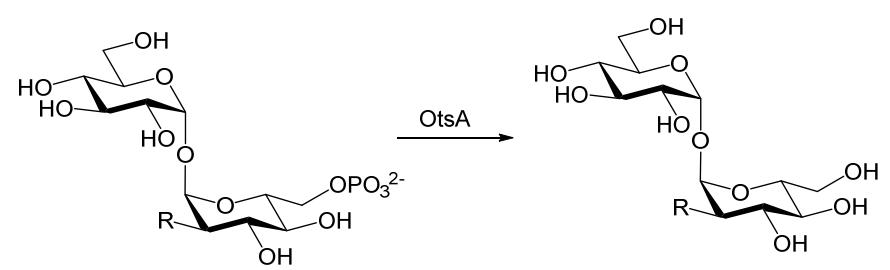

Scheme 24 Enzymatic synthesis of 2F-trehalose-6-phosphate using trehalose-6-phosphate synthase (OtsA) ${ }^{53}$ and of fluorinated trehaloses using trehalose synthase $(\text { TreT })^{54}$ 
In some cases, fluorinated glucose analogues have been used as an acceptor of transglycosidation by glycoside hydrolases (GHs). As mentioned earlier, synthetic activities of glycoside hydrolases are negatively offset by typically higher hydrolytic activity. ${ }^{23}$ To address this, Withers et al. introduced an idea of glycosynthase, engineering a glycoside hydrolase to remove a nucleophile residue, and thus making the mutant non-hydrolytic while the intact original structure of the mutant allows the formation of the glycosidic bond. ${ }^{55}$ In an early example of glycosynthase Abg E358A made from a glucosidase from Agrobacterium sp., $\alpha$-glucosyl fluoride was used as a donor and 2,4-dinitrophenly $\beta$-glucoside as an acceptor (Scheme 25). ${ }^{55}$ The reaction yielded a mixture of $\beta-(1 \rightarrow 4)$-linked di- (38\%), tri- $(42 \%)$ and tetrasaccharides $(4 \%)$. There are also examples that used wild type glycoside hydrolases in transglycosidation. $\alpha$-Glucosyl fluoride and $\alpha$-galactosyl fluoride were used to monitor transglycosidation kinetics of $\alpha$-glycoside hydrolases including $\alpha$-glucosidase from Baker's yeast Saccharomyces cerevisiae (Scheme 25) and $\alpha$-galactosidase from either green coffee beans or Bacillus stearothermophilus with ${ }^{19} \mathrm{~F}$ NMR.${ }^{56}$ These reactions yielded mixtures of anomeric fluorides of mostly $\alpha-(1 \rightarrow 2)$ and $\alpha-(1 \rightarrow 6)$ disaccharides. Field et al. also applied ${ }^{19} \mathrm{~F}$ NMR to monitor the transglycosidation activity of three GH enzymes, glucanotranferases from E. coli (EcMalQ), Manihot esculenta (MeDPE1) and Arabidopsis thaliana (AtDPE2) using 2-fluoro-, 3-fluoro- and 6-fluoro-glucose as an acceptor and glycogen as a donor (Scheme 25). ${ }^{57}$ These GHs are characterised by their high $\alpha-(1 \rightarrow 4)$ transglycosidation activity, thus given their name of glucanotransferase. All three fluorinated glucoses acted as an acceptor, yielding mixtures of maltooligosaccharides with different lengths in the reaction with the respective enzymes, and the conversion rates were $4-7 \%$ for $E c \mathrm{MalQ}, 12-17 \%$ for $M e \mathrm{DPE} 1$ and $15-28 \%$ for $A t \mathrm{DPE} 2$.

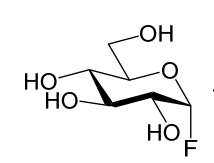

$\alpha \mathrm{GIcF}$

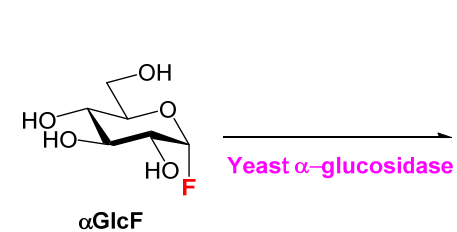

$\alpha \mathrm{GICF}$

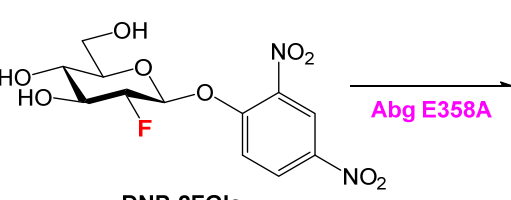

DNP-2FGIC
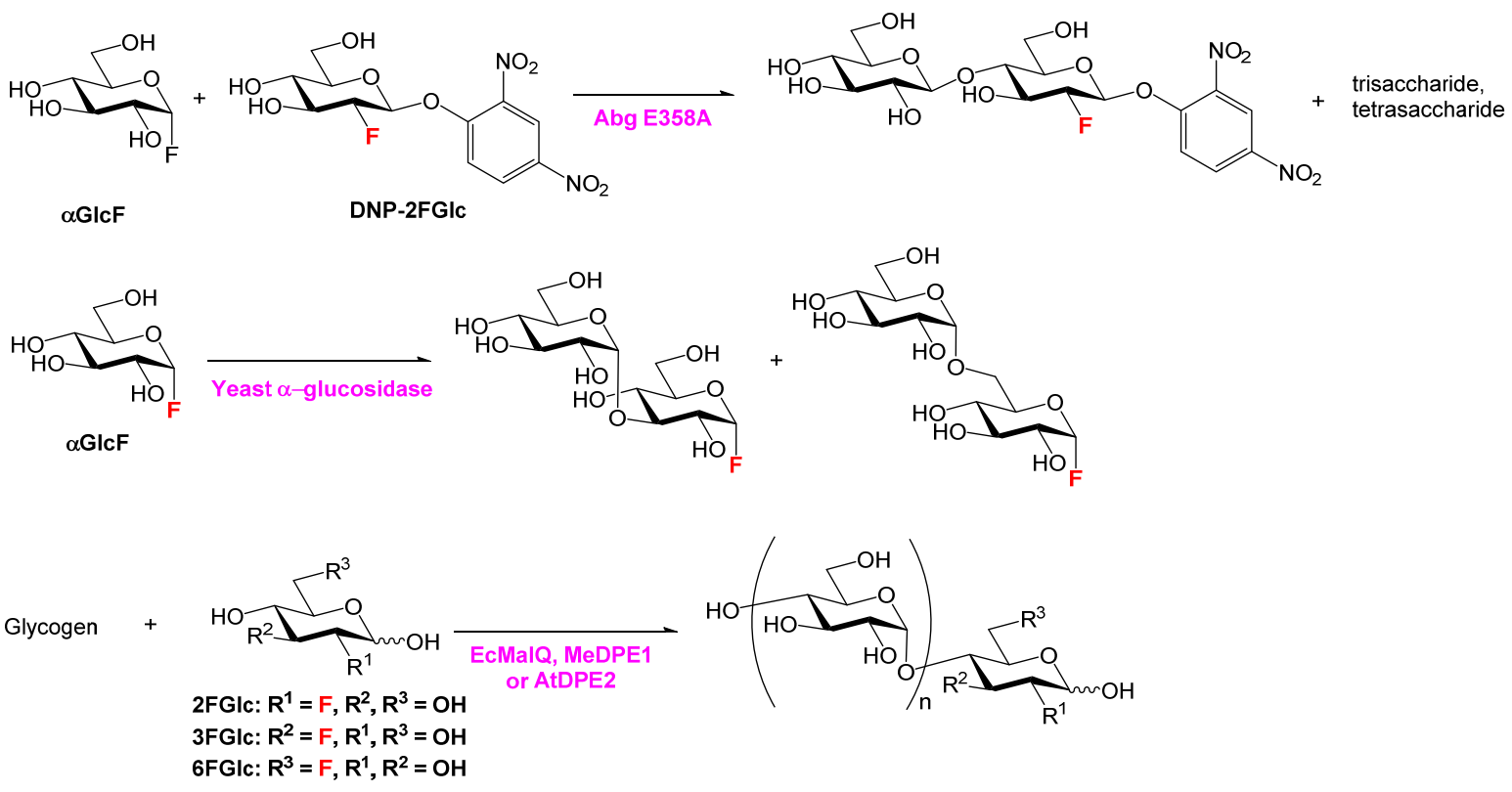
Scheme 25 Use of fluorinated glucose analogues as an acceptor of transglucosidation reaction catalysed by glycoside hydrolases. ${ }^{55-57}$

\section{Fluorinated $\mathrm{N}$-acetyl-galactosamine}

UDP-4FGalNAc is accessible from chemically synthesised 4FGalNAc-1P via a recombinant human enzyme, UDP-N-acetylglucosamine pyrophosphorylase (GlmU) (Scheme 26). Using a crude cell extract, an 18\% yield of UDP-4GalNAc was isolated and structurally characterised after $2 \mathrm{~h}^{58}$

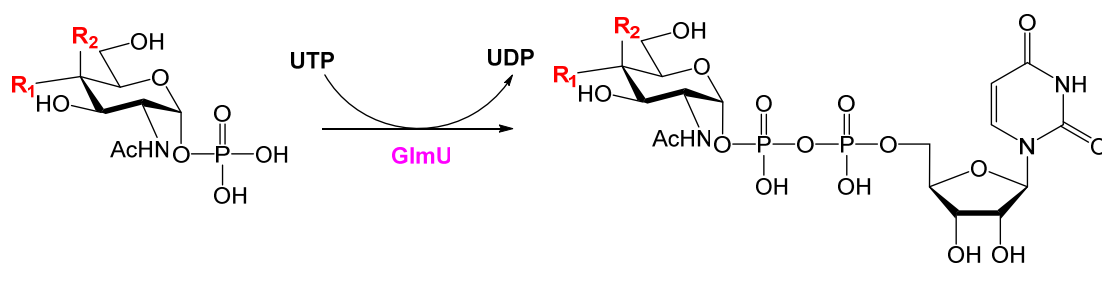

4FGICNAC $R_{1}=F, R_{2}=H \quad 23 \%$ 4FGalNAc $R_{1}=H, R_{2}=F 18 \%$

Scheme 26 Enzymatic synthesis of UDP-4FGalNAc and UDP-4FGlcNAc using human UDP-Nacetylglucosamine pyrophosphorylase $(\mathrm{GlmU}){ }^{58}$

Fluorinated GalNAc residues were also recognised as acceptor substrates for the phosphorylase enzyme BiGalHexNAcP (Scheme 27). The disaccharide Gal $\beta 1-3$ 6FGalNAc, with the fluorinated sugar moiety at the reducing end of the disaccharide can be obtained in milligram scale. The synthetic yield of $88 \%$ is in fact slightly higher than that with GalNAc as the acceptor (81\%). However the anomeric substituent in the GalNAc acceptor is $\mathrm{OH}$ and the increased yield may be a result of more facile purification owing to the azidopropyl group in the fluorinated acceptor rather than increased activity of the enzyme toward the acceptor. ${ }^{38}$

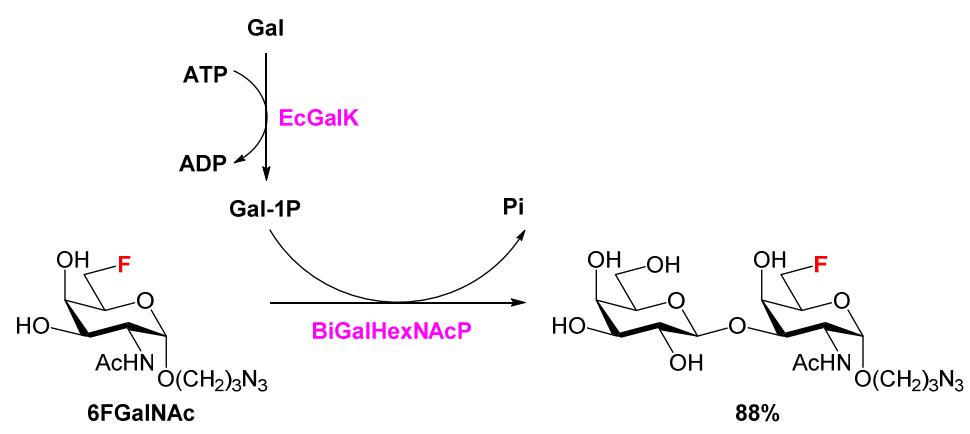


Scheme 27 Enzymatic synthesis of disaccharide Gal $\beta-(1 \rightarrow 3)$ 6FGalNAc using a phosphorylase, BiGalHexNAcP. ${ }^{38}$

\section{Fluorinated $\mathrm{N}$-acetyl-glucosamine}

Like UDP-4FGalNAc, UDP-4FGlcNAc can be synthesised by chemoenzymatic methods. Chemically synthesised 4FGlcNAc-1P can be converted enzymatically to UDP-4FGlcNAc using a recombinant human enzyme, GlmU (Scheme 26). ${ }^{58}$ Yields of UDP-4FGlcNAc are slightly better than the galacto-configured isomer ( $23 \%$ vs. $18 \%$ ) over shorter times ( $1 \mathrm{~h}$ vs. $2 \mathrm{~h}$ ), which is surprising given the natural substrate for the enzyme contains Gal rather than Glc stereochemistry.

Further to this, Amano et al. ${ }^{59}$ were able to demonstrate the in vivo synthesis of UDP-4FGlcNAc in a human cancer cell line (PC-3) from acetylated 4FGlcNAc sugars (the acetylation was required for cellular uptake) via hijacking of the cellular hexosamine biosynthetic pathway. Further studies on cellular metabolic glycans revealed that the 4FGlcNAc moiety was not incorporated in glycans produced by the Golgi- $N$-glycosylation pathways and instead UDP-4FGlcNAc acted as an inhibitor of GlcNAc transferases present in the pathway. 5F-GlcNAc derivatives were initially synthesised to probe whether the introduction of fluorine at the 5-position would render such compounds enzyme inhibitors. Preliminary studies indicated that 5FGlcNAc-1P is a substrate for the enzyme GlmU and could be used for the synthesis of UDP-5FGlcNAc (Scheme 28). ${ }^{60}$ UDP5FGlcNAc is however an inhibitor of the enzyme UDP-GlcNAc 4 epimerase ${ }^{60}$ and the glycosyltransferases, $\operatorname{CLS}^{61}$ and CgMshA. ${ }^{62}$
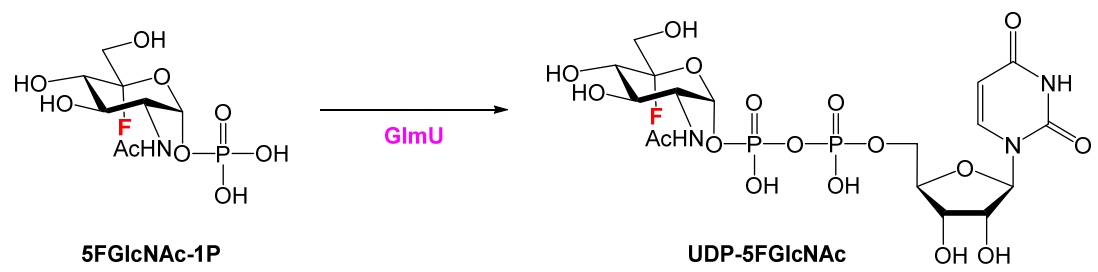

Scheme 28 Enzymatic synthesis of UDP-5FGlcNAc using GlmU. ${ }^{60}$

Conversely, the introduction of the fluorine at C-5 in acceptor substrates is recognised by the glycosyltransferases CLS and GalT (bovine milk), which transfer a galactosyl moiety to the 4-position, albeit at reduced efficiency when compared with the natural substrate GlcNAc for GalT (Scheme 29). ${ }^{60-61}$ Kinetic parameters suggest the low efficiency is due to a lower affinity of the enzyme for the modified acceptor, suggesting a large binding energy from 6'-OH of the acceptor. 


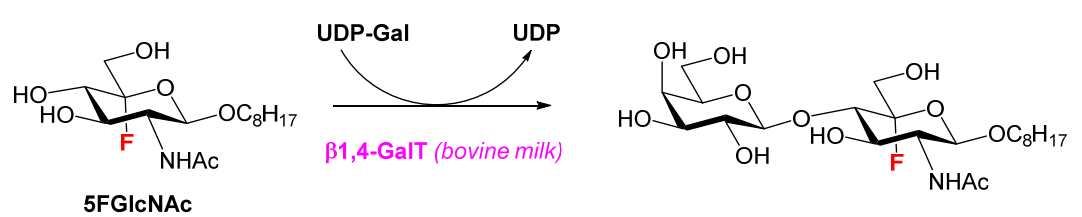

Scheme 29 Use of 5FGlcNAc as an acceptor of bovine milk $\beta$-1,4-galactosyltransferase. ${ }^{60}$

A different type of fluorination, not directly part of the pyranose ring, but at the $N$-acetyl group, is possible in DGlcNAc. In an early study, it was demonstrated that chemically synthesized UDP-N-trifluoroacetylglucosamine (UDP-GlcNTFA) was tolerated fairly well by two glucosaminyltransferases, 'core-2' GlcNAc transferase, involved in the biosynthesis of O-linked glycoproteins, and UDP-D-GlcpNAc: $\alpha-D-M a n p-(1 \rightarrow 6)-\beta-D-G l c p N A c$ transferase V (GlcNAcT-V), a key biosynthetic enzyme controlling the branching pattern of cell surface complex Asn-linked oligosaccharides (Scheme 30). ${ }^{63}$ Both enzymes were able to synthesize trisaccharide products with $62 \%$ and $56 \%$ isolated yield. Later, it was found that UDP-GlcNTFA was an excellent substrate for Pasteurella multocida strain P-1059 heparosan synthase 2 (PmHS2), a bifunctional enzyme that acts both as $\alpha$-1,4- $N$-acetylglucosaminyltransferase and $\beta$-1,4-glucuronyltransferase, and Escherichia coli K5 $\alpha 1$ -

4GlcNAcT. ${ }^{64-65}$
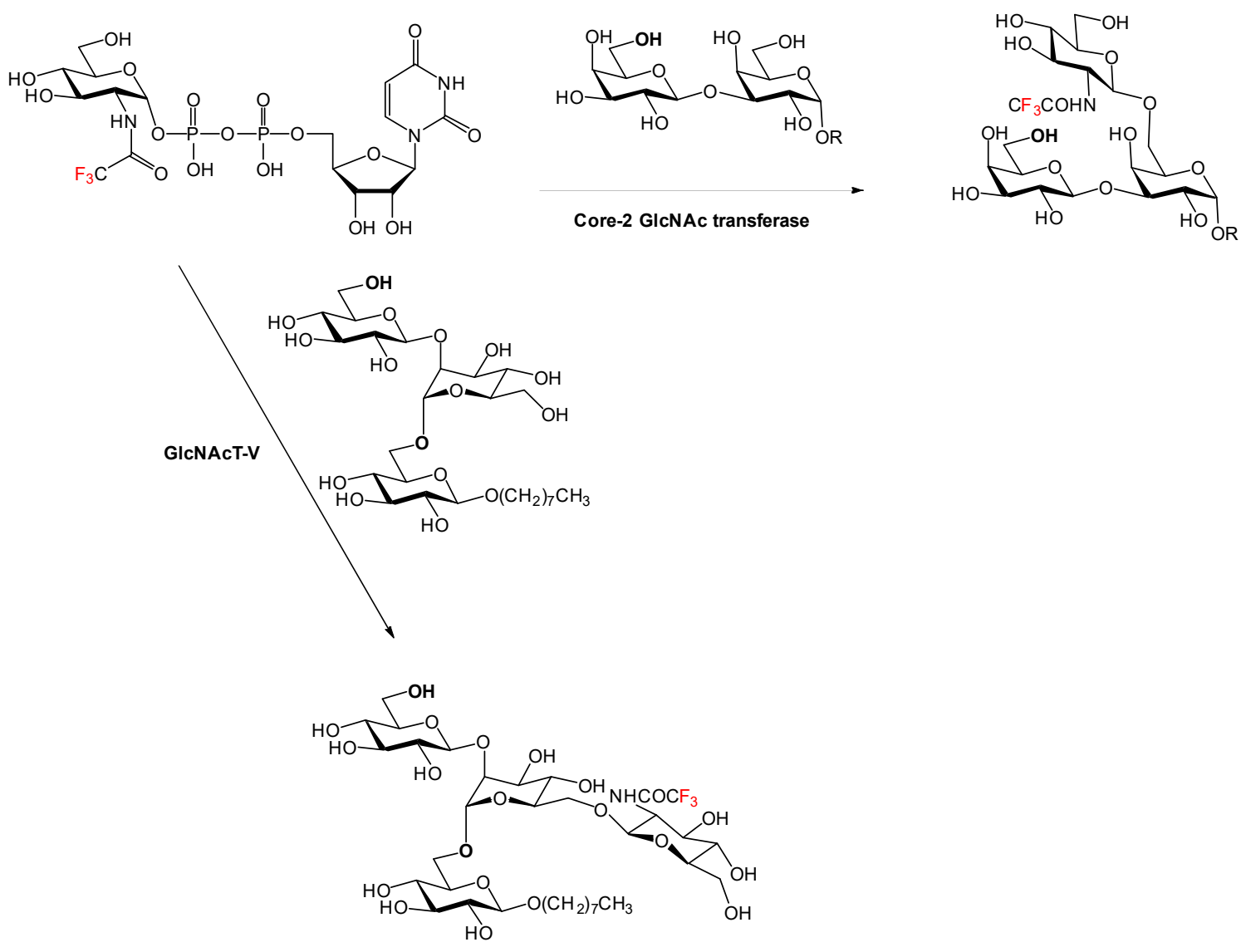
Scheme 30 Use of UDP-GlcNTFA as a donor of N-acetylglucosaminyltransferases, Core-2 GlcNAc transferase and GlcNAcT-V. ${ }^{63}$

It was also demonstrated that the acceptor UDP-GlcNTFA could be synthesized enzymatically using Bifidobacterium longum ATCC55813 N-acetylhexosamine 1-kinase (NahK) and Pasteurella multocida strain P1059 (ATCC15742) $N$-acetylglucosamine-1-phosphate uridylyltransferase (PmGlmU). ${ }^{64,66}$ Along with these two enzymes, PmHS2 was used in a one-pot multi-enzyme system for the synthesis of heparan sulfate oligosaccharides (Scheme 31). The trifluoroacetyl moiety was then hydrolysed in a mild basic condition, and the resulting free amine moiety sulfated either enzymatically or chemically, yielding natural heparan sulfate oligosaccharides. ${ }^{67}$
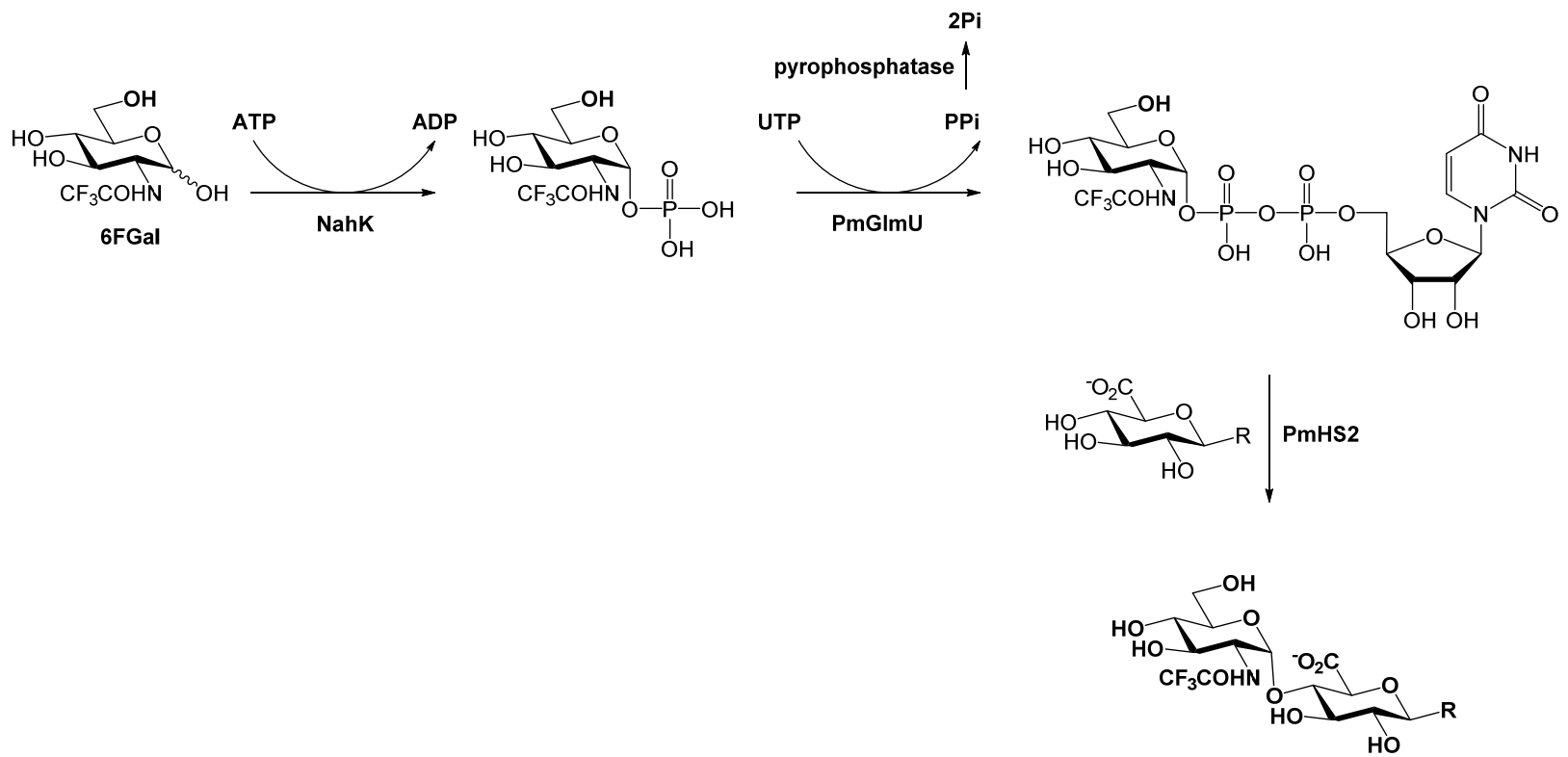

Scheme 31 One-pot multi-enzyme system for the synthesis of heparin sulfate oligosaccharides containing NahK (N-acetylhexosamine 1-kinase), PmGlmU (Pasteurella multocida strain P-1059 (ATCC15742) Nacetylglucosamine-1-phosphate uridylyltransferase), inorganic pyrophosphatase, and PmHS2 (heparosan synthase 2 , bifunctional glycosyltransferase) ${ }^{64}$

\section{Fluorinated Talose}

In addition to tolerating all the four isomers of FGal-1P, GalPUT is also able to process sugars which contain the talose stereochemistry (axial at C-2). Errey and co-workers demonstrated that the immobilised system described in section 3.1 is able to synthesise UDP-2FTal from 2FTal in 71\% yield (Scheme 32 ). ${ }^{68}$ This is greater 
than for unmodified Tal (15\%) and although yields are comparable to Gal and derivatives, longer reaction times ( $24 \mathrm{~h} v s .8 \mathrm{~h})$ are needed.
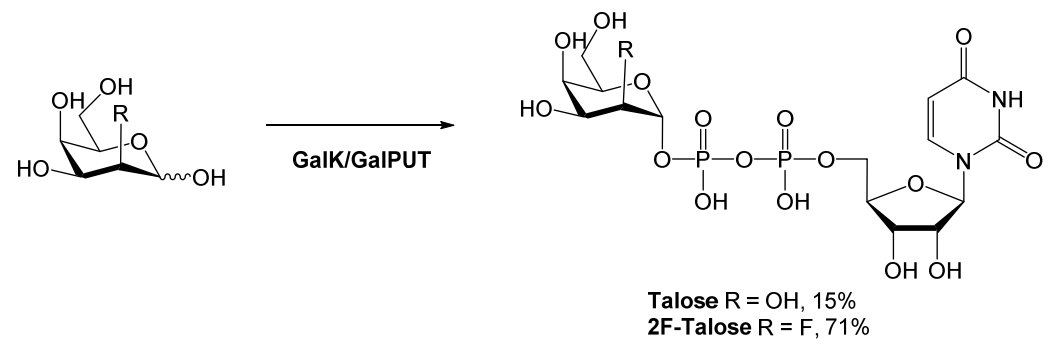

Scheme 32 Enzymatic synthesis of UDP-2F-talose using the GalK/GalPUT system. ${ }^{68}$

\section{Fluorinated Fucose}

2-Deoxy-2-fluorinated analogues of sialyl Lewis a can be synthesised in milligram quantities from the donor GDP-2F-L-Fuc using the fucosyltransferase (FucT) III enzyme (Scheme 33) ${ }^{69}$ Extended reaction times (up to one day) are needed to achieve a comparable yield (88\%) to the natural donor GDP-L-Fuc (97\%). However, the stereo- and regio-selectivity of the enzyme is not altered by the fluorinated donor. This result is remarkable since fluorination at the position adjacent to the anomeric centre normally creates a highly unfavourable environment for the glycosyl transfer reaction that involves an oxocarbenium ion-like transition state. ${ }^{70}$ Usually, GDP-2F-L-Fuc has been observed to act as a competitive inhibitor of a number of human fucosyltransferases.

${ }^{71}$ In line with this, Fuc-T VI does not accept GDP-2FFuc to synthesise sialyl Lewis $\mathrm{x}$ analogues ${ }^{69}$ although the authors did not evaluate the fluorinated analogue as an inhibitor.
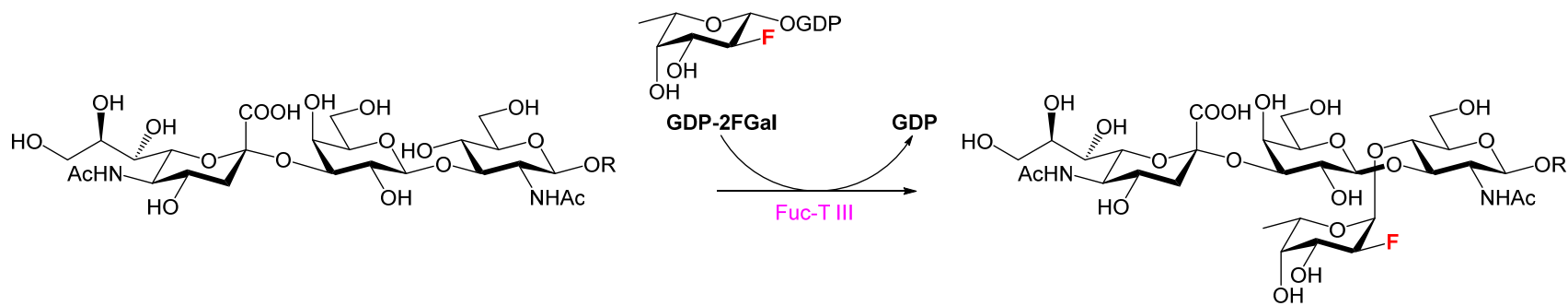

Scheme 33 Enzymatic synthesis of sialyl Lewis a containing 2-fluoro-L-fucose. ${ }^{69}$

GDP-6F-L-Fuc has also been shown to be a competitive inhibitor toward the human fucosyltransferases III, V, VI and VII , however the 6F-L-Fuc moiety can be introduced into Lewis $\mathrm{x}$ analogues from 6F-L-Fuc making use of the bifunctional enzyme L-fucokinase/GDP-fucose pyrophosphorylase (FKP) and the bacterial $\alpha-(1 \rightarrow 3)$ fucosyltransferase (Scheme 34). ${ }^{72}$ Although FKP has a lower affinity for 6F-L-Fuc than L-Fuc, milligram quantities of GDP-6F-L-Fuc can be prepared in 94\% yield from 6FFuc in 5-6 hours. When FKP is combined in 
a one-pot, two-enzyme reaction with the fucosyltransferase the sialyl Lewis $\mathrm{x}$ derivative can be synthesised directly from 6F-L-Fuc, without the need to isolate GDP-6F-L-Fuc, in 90\% yield. In both cases, the regio- and stereoselectivity of the enzymes was confirmed spectroscopically.
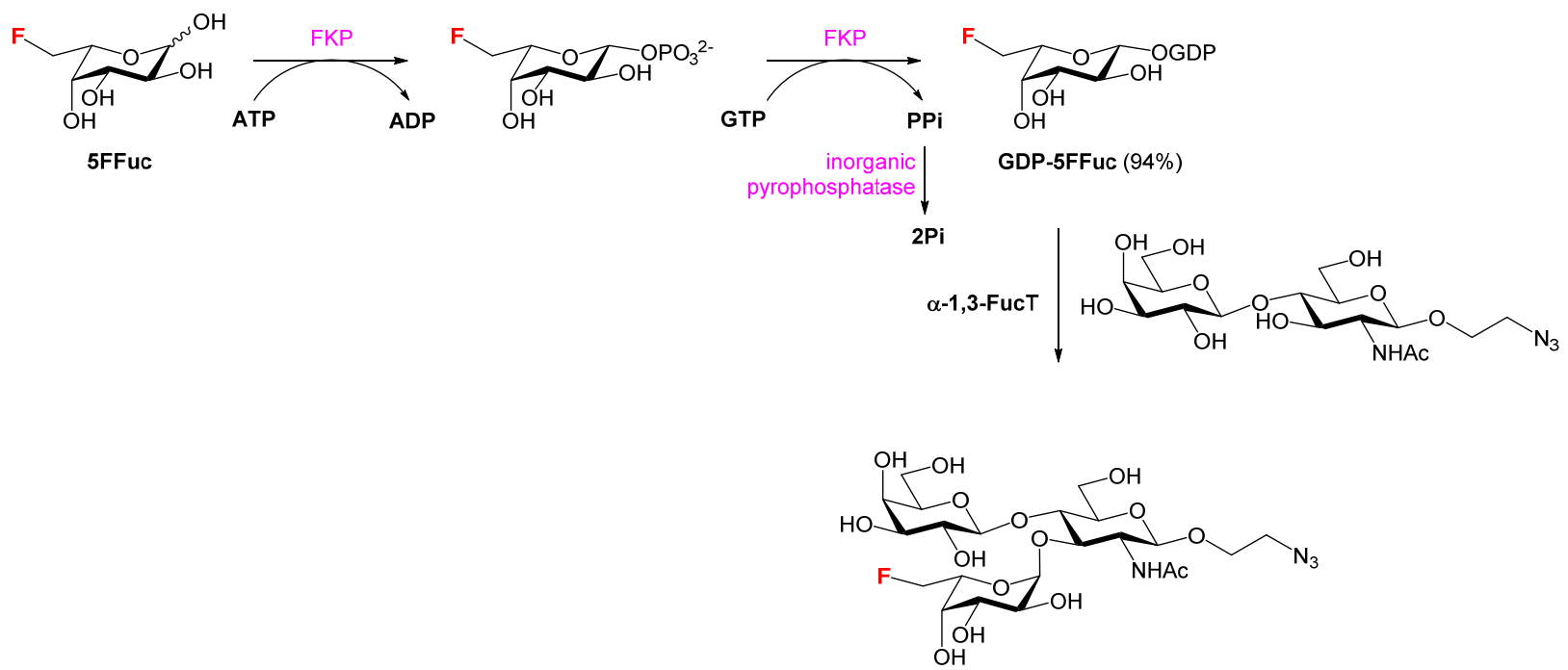

Scheme 34 Enzymatic synthesis of Lewis x derivative containing 6-fluoro-L-fucose. ${ }^{72}$

When combined with the $\alpha-(1 \rightarrow 2)$ fucosyltransferase FutC, FKP can also append a 6F-L-Fuc moiety to the nonreducing end of a Gb5 oligosaccharide to yield a derivative of Globo H (Scheme 35). Although FKP can be used to synthesise GDP-gem-6FF-Fuc from gem-6FF-Fuc, the former is not a good substrate for FutC, with only trace amounts of product formed..$^{73}$

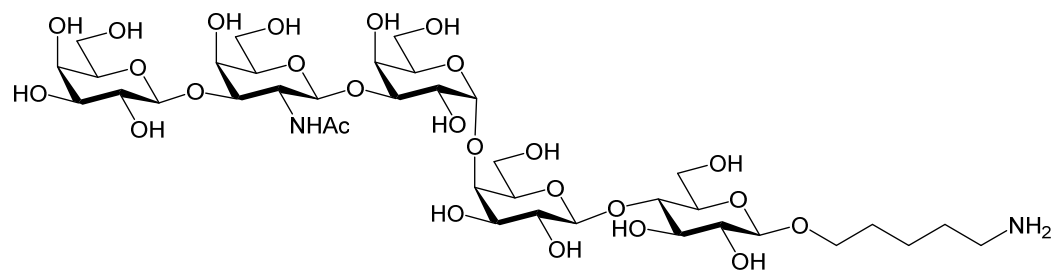

Gb5

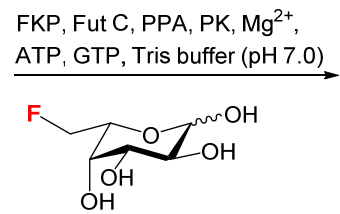

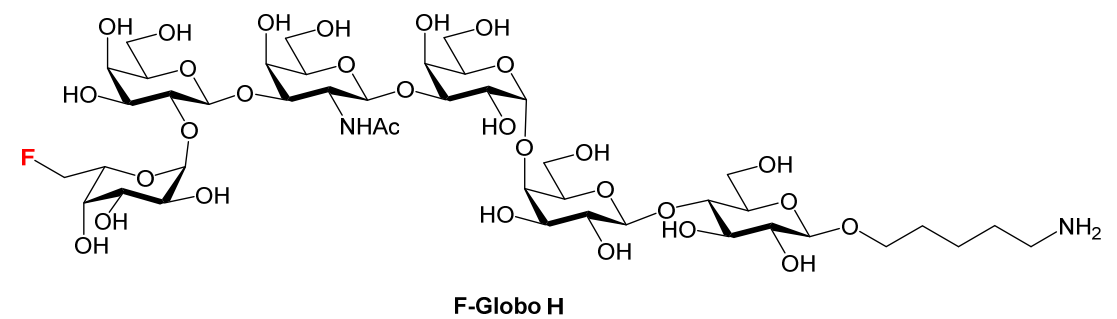


Scheme 35 One-pot multi-enzyme system for the synthesis of fluorinated derivative of Globo-H. ${ }^{73}$ FKP $=\mathrm{L}-$ fucokinase/GDP-fucose pyrophosphorylase, PPA - inorganic pyrophosphatase, Fut C $=\alpha-1,2-$ fucosyltransferase.

\section{Fluorinated Sialic Acids}

Some fluorinated sialic acid donors have been shown to inhibit SiaTs or TcTS, whilst others have been shown to act as substrates for sialic acid processing enzymes, many of which are discussed in the following section.

\subsection{3-Fluorinated sialic acids}

Watts and Withers demonstrated that CMP-3F $F^{\text {ax }}$ Neu5Ac could be synthesised in $91 \%$ yield from $3 \mathrm{~F}^{\mathrm{ax}} \mathrm{Neu} 5 \mathrm{Ac}$ and CTP using CMP-sialic acid synthase (CSS). To effect this transformation, the authors used a sialic acid aldolase (Scheme 36) ${ }^{74}$ In this synthesis sialic acid aldolase catalyses the formation of Neu5Ac from ManNAc and pyruvate. ${ }^{75}$ By using fluoropyruvate in place of pyruvate, $3 \mathrm{~F}^{\mathrm{ax}} \mathrm{Neu} 5 \mathrm{Ac}$ was successfully synthesised. When the two enzyme reactions were coupled together, CMP-3F ${ }^{a x}$ Neu5Ac was isolated in $84 \%$ yield over 2 steps from ManNAc. Interestingly, under these conditions only the isomer bearing the 3-fluorine in the axial position was obtained. ${ }^{74}$
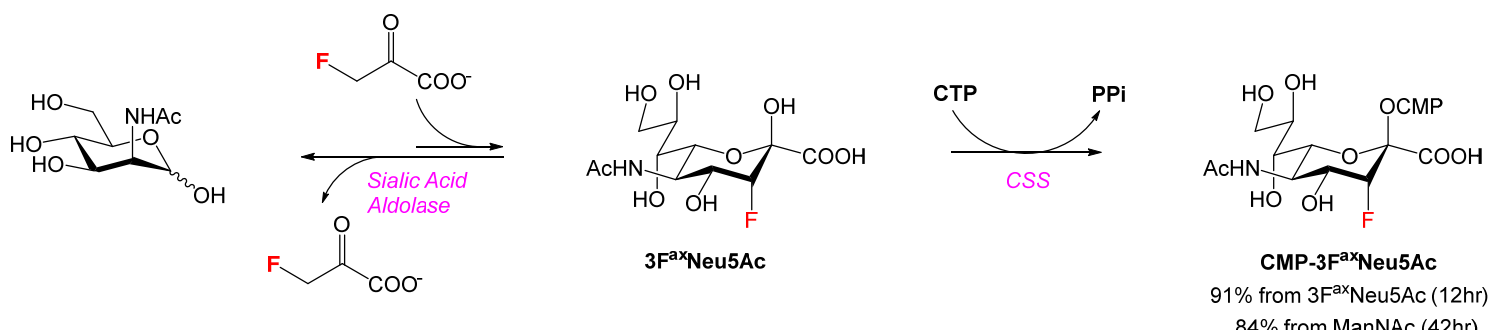

Scheme 36 The two step enzymatic synthesis of CMP-3Fax Neu5Ac. ${ }^{74}$

Chen et al. ${ }^{76}$ later demonstrated that when the condensation was carried out with a recombinant aldolase from $E$. coli a mixture of both $3 \mathrm{~F}$ axial and equatorial isomers were formed. These isomers could be which readily separated by flash chromatography and isolated in in 52 and $44 \%$ yield, respectively. Axial and equatorial isomers of 3-fluoro- $N$-glycolylneuraminic acid (3FNeu5Gc) and 3-fluoro-keto-deoxynonulosonic acid (3FKDN) 
were also formed from the aldolase promoted condensation of 3-fluoropyruvate and N-glycolylmannosamine (ManNGc) or Man respectively.

Despite early claims that neither $3 \mathrm{~F}^{\mathrm{eq}} \mathrm{Neu} 5 \mathrm{Ac}$ nor $3 \mathrm{~F}^{\mathrm{ax}} \mathrm{Neu} 5 \mathrm{Ac}$ are substrates for CSS from calf brain ${ }^{77,78}$, Chen and colleagues went on to demonstrate the both $3 \mathrm{~F}^{\mathrm{ax}} \mathrm{Neu} 5 \mathrm{Ac}$ and $3 \mathrm{~F}^{\mathrm{eq}} \mathrm{Neu} 5 \mathrm{Ac}$ were substrates for CSS from Neisseria meningitides (NmCSS), and the CMP-sialic acids could be synthesised in excellent yields (Scheme $37)^{76}$. Furthermore, Heise et al. reported that a C-5 carbamate increases the efficiency of the conversion of $3 \mathrm{~F}$ sialic acids to the relevant CMP analogue. ${ }^{79}$
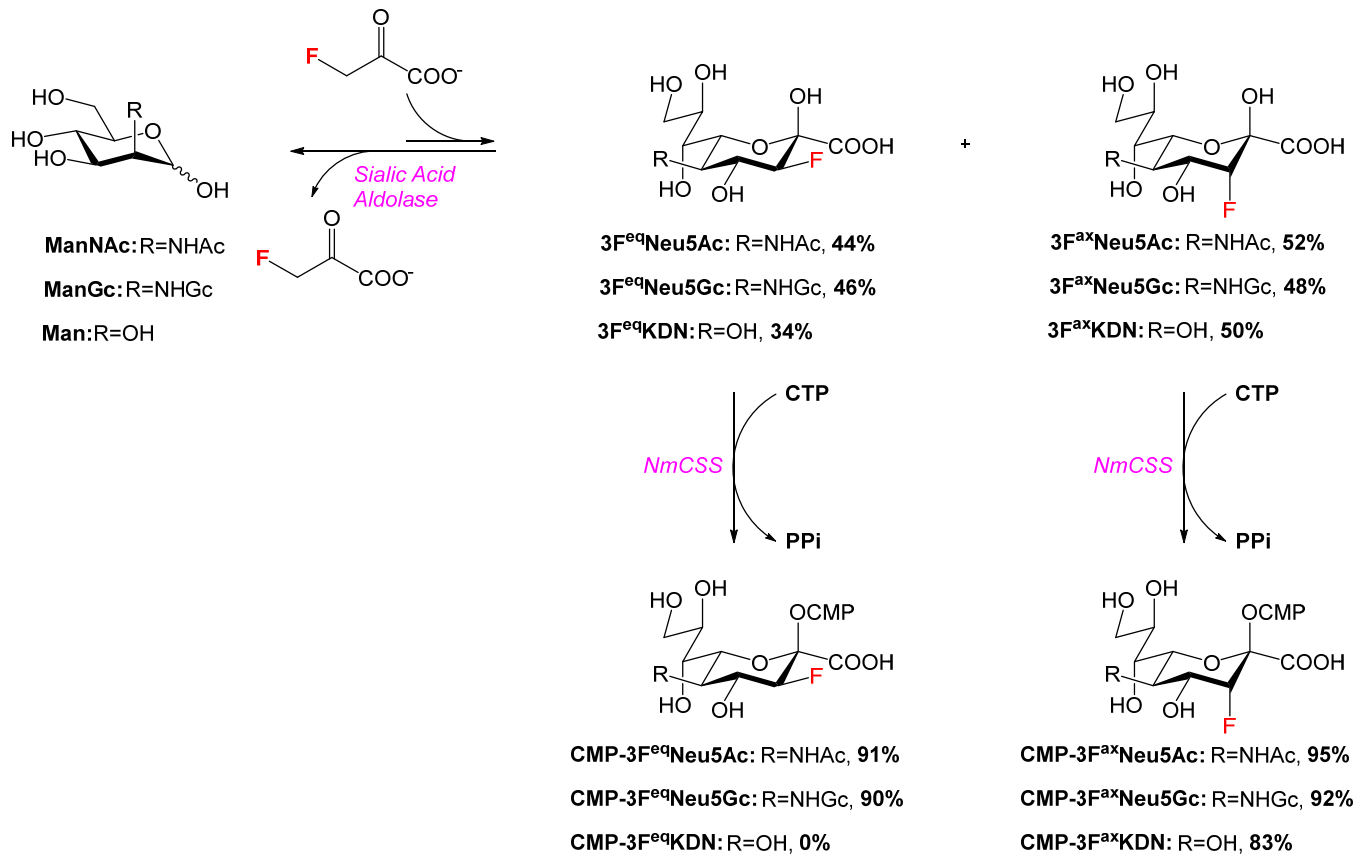

Scheme 37 The enzymatic synthesis of CMP activated 3F sialic acid analogues. ${ }^{76}$

The presence of the electronegative fluorine atom adjacent to the anomeric carbon has meant that CMP$3 \mathrm{~F}^{\mathrm{ax}} \mathrm{NeuAc}$ has been utilised as an inhibitor of a commercial $\alpha-(2 \rightarrow 6) \mathrm{SiaT}^{78}$ the SiaT Cst-II from Campylobacter jejuni ${ }^{80}$ and TcTs. ${ }^{81}$ Nevertheless, Chen et al. demonstrated that CMP-3F ${ }^{\text {ax }}$ Neu5Ac and CMP-

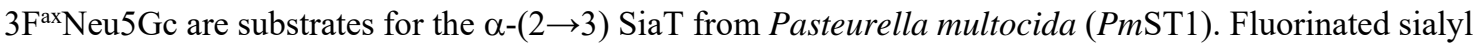
lactosides can be prepared in a one-pot, two-enzyme (NmCSS and PmST1) system from the corresponding 3F $\mathrm{F}^{\mathrm{ax}}$ sialic acid (Scheme 38). Under the same conditions, the sialyl lactosides containing the $3 \mathrm{~F}^{\mathrm{eq}}$ sialic acid could be obtained in higher yields. The $\alpha-(2 \rightarrow 6)$ SiaT from Photobacterium damselae $(P d 2,6 \mathrm{ST})$ does not appear to 
tolerate the fluorinated donors as well as PmST1 can, and corresponding yields of the $\alpha 2-6$ fluorosialyl lactosides were much lower. ${ }^{76}$
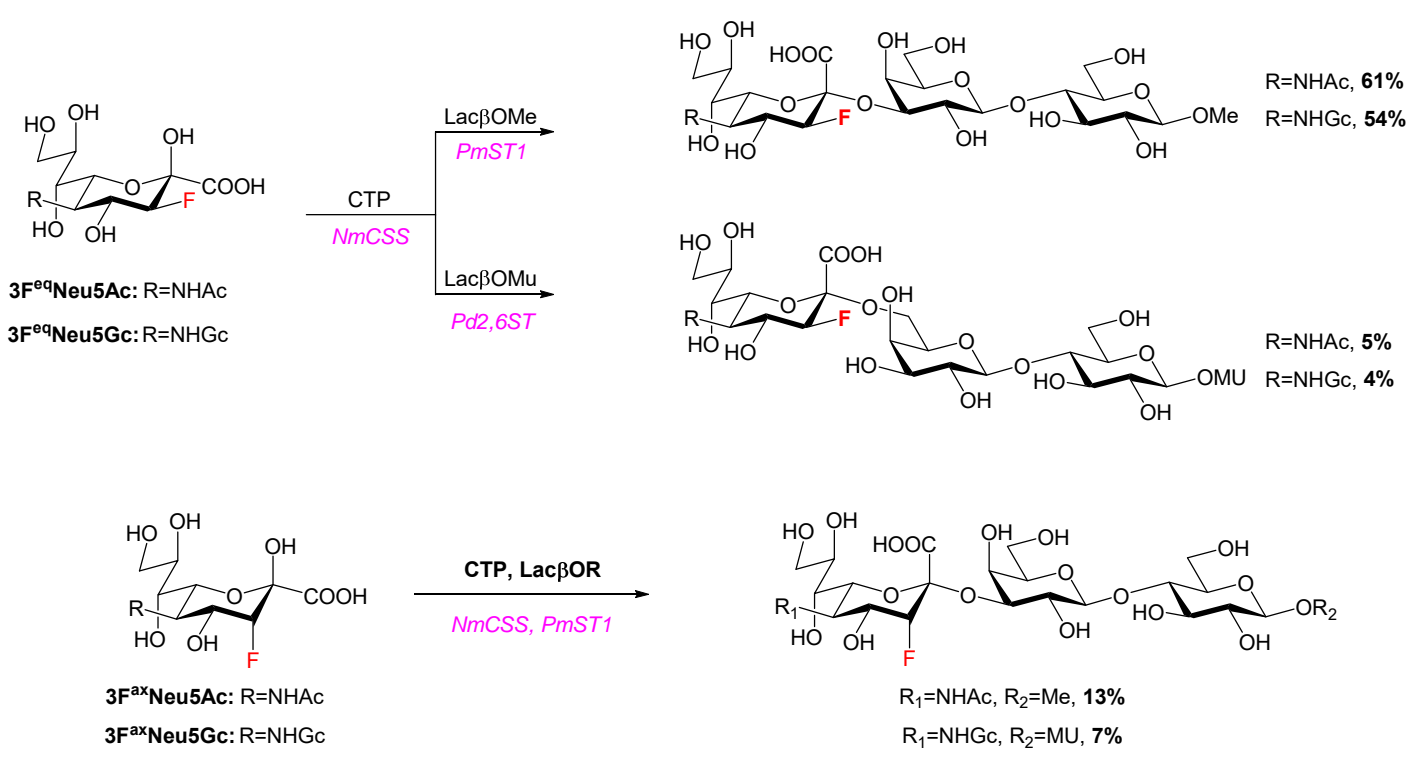

Scheme 38 The one-pot, two enzyme synthesis of $3 F$ sialic acid-containing trisaccharides. ${ }^{76}$

\subsection{5-Fluorinated sialic acids}

Wong et al. ${ }^{82}$ reported the synthesis of 5-fluorinated 5-epi KDN in 30\% yield from 2-deoxy-2-fluoroglucose (2FGlc) and pyruvate using a sialic acid aldolase (Scheme 39). However, microbial CSS did not recognise 5F, 5-epi KDN as a substrate, possibly due to either the lack of 5-NHAc, or the differing stereochemistry (axial) at C-5 compared with Neu5Ac or KDN (equatorial).

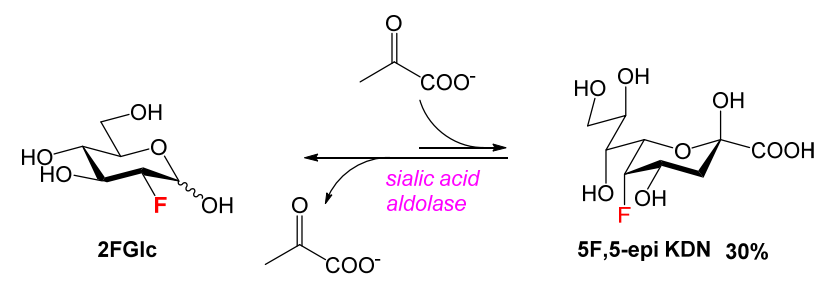

Scheme 39 The enzymatic synthesis of 5, 5-epi FKDN from 2FGIc. ${ }^{82}$

More recently, Chen et al. ${ }^{83}$ reported the enzymatic synthesis of 5FKDN from 2-deoxy-2-fluoromannose (2FMan) using a sialic acid aldolase from Pasteurella multocida (Pm sialic acid aldolase). The authors utilised this transformation in a one-pot, three-enzyme system which also contained NmCSS and either PmST1 or $P d 2,6 \mathrm{ST}$ with a para-nitrophenyl galactoside (Gal $\beta p N P$ ) acceptor to give either the $\alpha 2-3$ or $\alpha 2-6$ fluorinated 
disaccharide in yields of $74 \%$ and $79 \%$ respectively (Scheme 40 ). Despite these good yields, they were not as high as for the analogous reactions containing Neu5Gc derivatives.
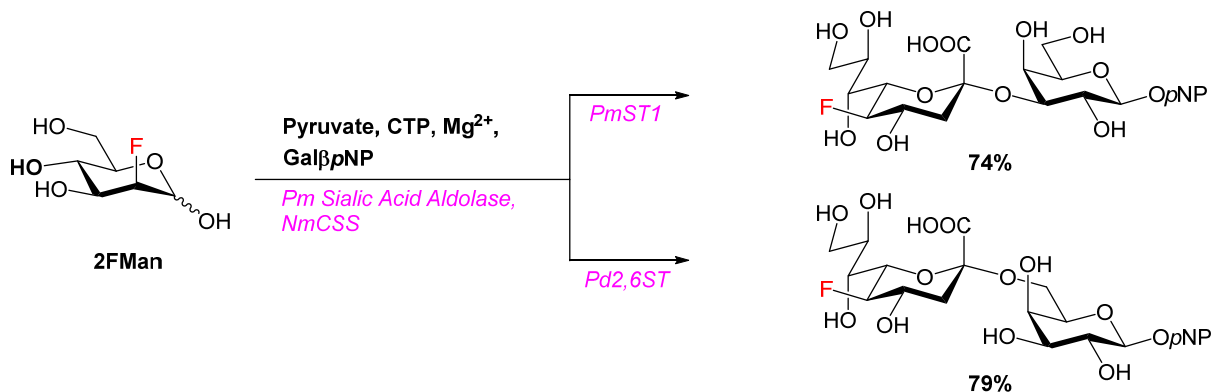

Scheme 40 The one-pot, three-enzyme synthesis of 5FKDN-containing disaccharides. ${ }^{83}$

\subsection{7-Deoxy-7-fluoro sialic acids}

The enzymatic synthesis of CMP-7FNeu5Ac from the corresponding 4FManNAc using sialic acid aldolase and CSS was initially explored by Dräger et al. ${ }^{84}$ Using conditions which had resulted in full conversion of ManNAc to Neu5Ac, only $56 \%$ conversion to $7 \mathrm{FNeu} 5 \mathrm{Ac}$ was obtained from $4 \mathrm{FManNAc}$ using a commercially available aldolase enzyme (Scheme 41). In order to shift the equilibrium of the aldolase catalysed reaction toward the sialic acid, coupling with the CSS from Neisseria meningitidis serogroup B (NmBCSS) was explored. This approach, however, did not significantly improve conversion to CMP-7FNeuAc as determined by LC-ESI-MS and both 4FManNAc and 7FNeu5Ac remained. Full conversion to CMP-7FNeuAc was only achieved when isolated 7FNeu5Ac was turned over by NmBCSS in the presence of pyrophosphatase. It is worthy to note that the authors investigated other sources of CSS (murine CSS or rainbow trout CMP-Kdnsynthetase), however best results were obtained with NmBCSS.

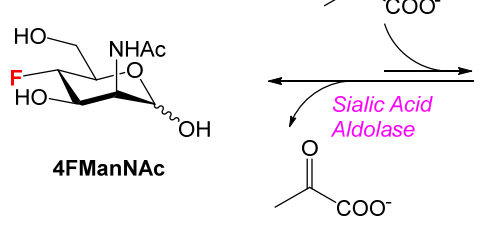

Scheme 41 The enzymatic synthesis of CMP-7FNeu5Ac. ${ }^{84}$

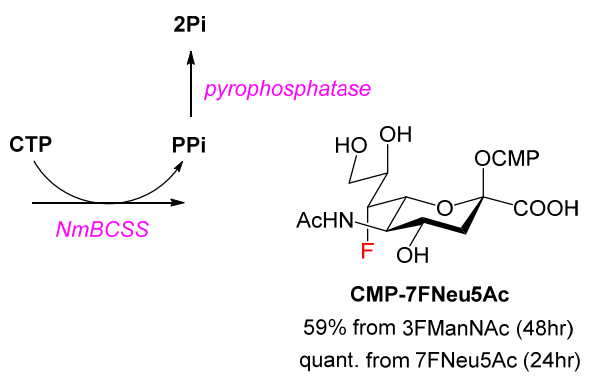

Preparative scale synthesis of sialosides containing the 7FNeu5Ac from hexoses via a one-pot, three-enzyme system was later demonstrated by Khedri et al. ${ }^{85}$ Using Gal $\beta p N P$ as an acceptor and employing Pm sialic acid aldolase, NmCSS and either PmST1 or Pd2,6ST, milligram scale synthesis of the galactosyl $\alpha-(2 \rightarrow 3)$ or $\alpha$ - 
$(2 \rightarrow 6)$ sialosides could be isolated and fully characterised in 98 and $92 \%$ yields respectively (Scheme 42).

Using the same enzymes, the $\alpha-(2 \rightarrow 3)$ and $\alpha-(2 \rightarrow 6) 7 F K D N$ disaccharides could also be synthesised from 4FMan in 98 and 95\% yields respectively. In both cases, isolation of the sialic acid or CMP-sialic acid was not required, and the stereo- and regioselectivity of the SiaTs was not altered with the fluorinated sugar.
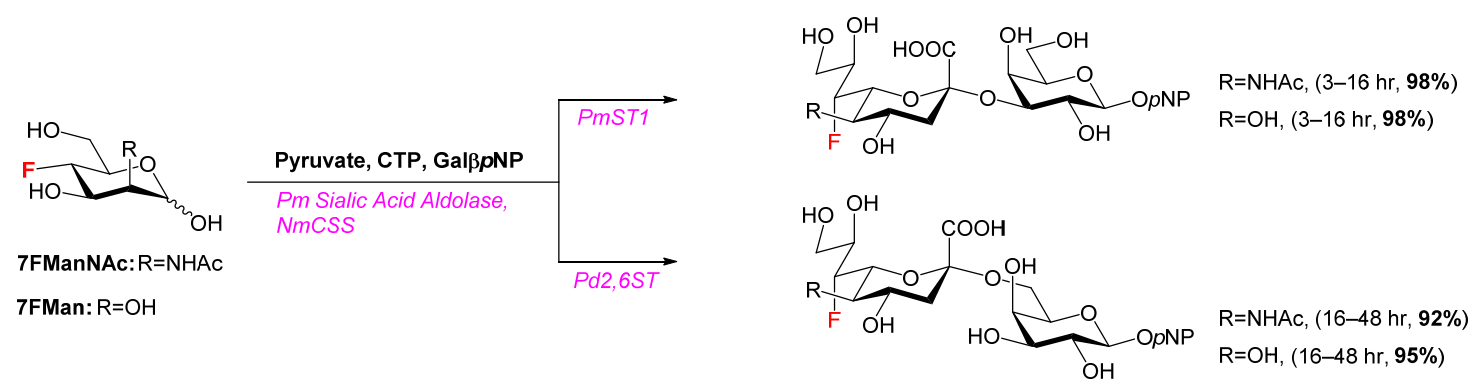

Scheme 42 The one-pot, three enzyme system used to synthesise disaccharides containing 7F sialic acid moieties. ${ }^{85}$

\subsection{8-Deoxy-8-fluorosialic acids}

CMP-8FNeu5Ac can be isolated in 82\% yield from 8FNeu5Ac after 6 hours reaction with NmCSS and an inorganic pyrophosphatase (Scheme 43). Kinetic studies revealed that the $\alpha-(2 \rightarrow 3)$ SiaT from Campylobacter jejuni (Cst-I) is able to transfer CMP-8FNeu5Ac to modified lactose acceptors (60 minutes), albeit with less efficiency than the natural donor CMP-Neu5Ac (2 minutes). Nonetheless, a sialyl lactose derivative could still be isolated in milligram quantities and $80 \%$ yield using this enzyme. Spectroscopic analysis confirmed the regio- and stereoselectivity of the enzyme was retained with the fluorinated donor. Under conditions optimal for $\alpha-(2 \rightarrow 3)$-sialyltransfer activity, the multifunctional enzyme Pm0188h (Pasteurella multocida) is also able to transfer CMP-8FNeu5Ac to a modified lactose acceptor, but unlike Cst-I the reaction does not proceed to completion, possibly due to hydrolysis of the sialylated product ${ }^{86}$ Indeed, the authors acknowledge Pm0188h has been shown to exhibit $\alpha 2,3$-sialidase activity as well as $\alpha 2,3$-trans-sialidase and $\alpha 2,6$-sialyltransferase behaviour. ${ }^{86-87}$ 

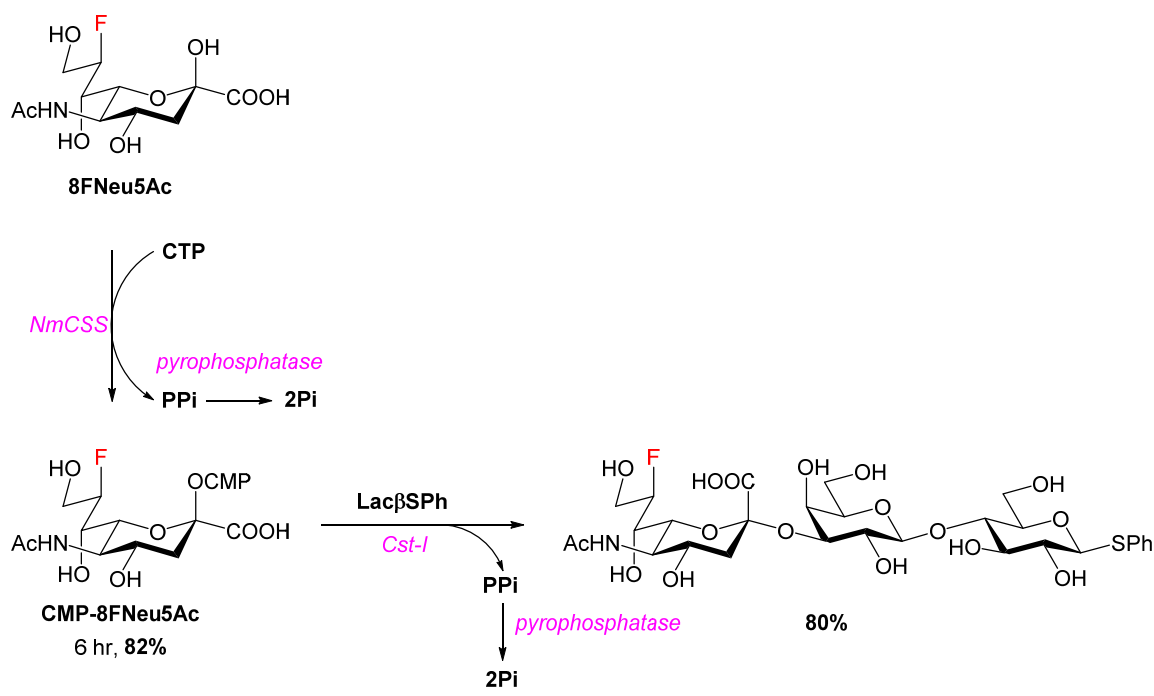

Scheme 43 The enzymatic synthesis of an $8 \mathrm{~F}$ sialyl lactose derivative. ${ }^{86}$

\subsection{9-Deoxy-9-fluorosialic acids}

Early analytical studies suggested that chemically synthesised 9F-Neu5Ac was a substrate for CSS from calf brains, albeit kinetics showed the enzyme displayed a lower affinity toward the fluorinated substrate compared for the natural substrate Neu5Ac..$^{77,88}$

9F-Neu5Ac has been synthesised enzymatically from 6FManNAc, and ${ }^{14} \mathrm{C}$-radio-labelling was introduced at the 2-position by Conradt et al. via the coupling of $6 \mathrm{FManNAc}$ and $\left[2-{ }^{14} \mathrm{C}\right]$ pyruvic acid using the enzyme acylneuraminate pyruvate-lyase from Clostridium perfringens with an overall yield of 60\% (Scheme 44).

Although kinetic analysis revealed a higher $\mathrm{Km}$ for $9 \mathrm{~F}\left[2-{ }^{14} \mathrm{C}\right] \mathrm{Neu} 5 \mathrm{Ac}$ than that of the natural substrate Neu5Ac, activation of the fluorinated substrate with CSS from rat liver gave CMP-9F $\left[2-{ }^{14} \mathrm{C}\right] \mathrm{Neu} 5 \mathrm{Ac}$ in $30 \%$ yield after purification. The authors went on to demonstrate CMP-9F $\left[2-{ }^{14} \mathrm{C}\right] \mathrm{Neu} 5 \mathrm{Ac}$ is a substrate for an $\alpha-(2 \rightarrow 6)$ SiaT from rat liver, and can be transferred onto a galactosyl terminated asialo- $\alpha 1$-acid glycoprotein acceptor, albeit at a lower rate than CMP-Neu5Ac even at higher sugar nucleotide concentrations. ${ }^{89}$ 

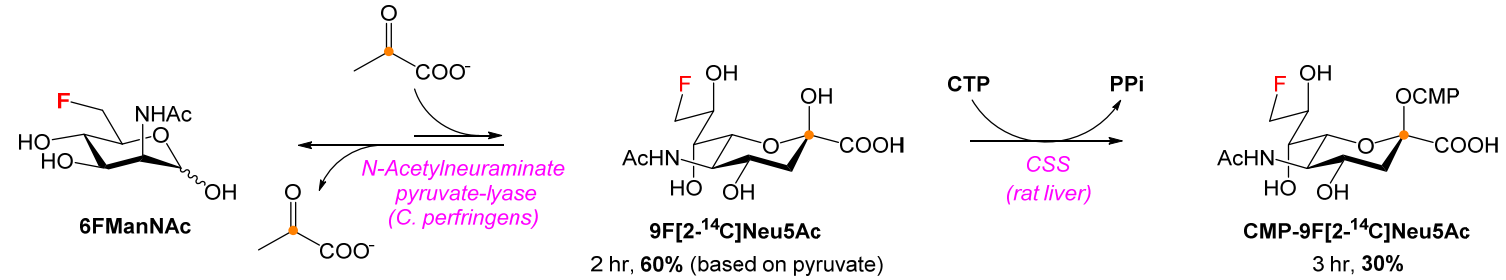

Scheme 44 The enzymatic synthesis of CMP-9F $\left[2-{ }^{14} \mathrm{C}\right]$ Neu5Ac, orange dot denotes the position of the isotopic label. ${ }^{89}$

Liu et al..$^{82}$ have also demonstrated the synthesis of 9-deoxy-9-fluorinated sialic acid derivatives of Neu5Ac and KDN from modified hexopyranose derivatives and pyruvate using sialic acid aldolase (Scheme 45). Kinetic studies revealed that CSS enzymes from E. coli (both a tagged and native enzyme) were able to turnover 9FNeu5Ac with parameters similar to those for the natural substrate Neu5Ac. Conversely, the 7F, 9F difluorinated 7-epi KDN derivative was not recognised by the enzyme, however the authors postulate this observation was due to the lack of a 5-NHAc group rather than the presence of the fluorine.

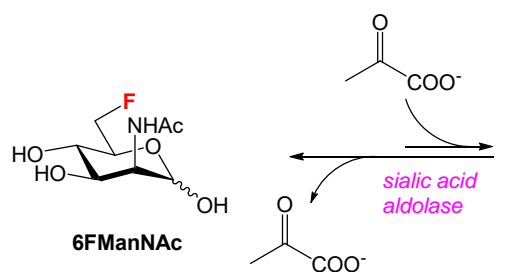

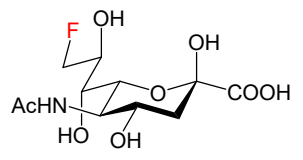

9FNeu5Ac 8 days, $22 \%$
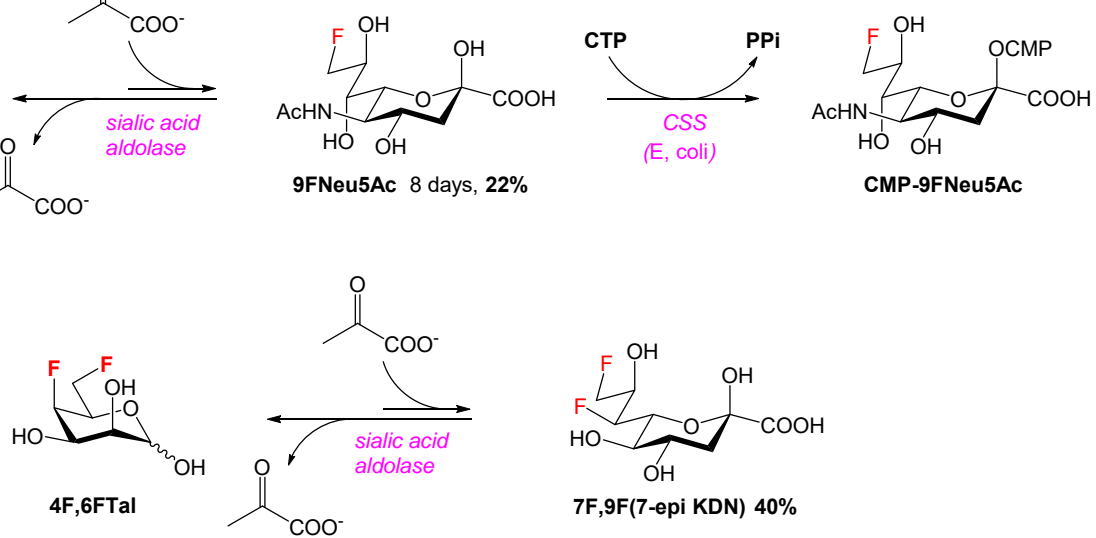

Scheme 45 The enzymatic synthesis of $9 \mathrm{~F}$ derivatives of Neu5Ac and 7-epi KDN. 82

Using the same one-pot, three-enzyme system described for the synthesis of 7 -fluorinated $\alpha-(2 \rightarrow 3)$ or $\alpha-(2 \rightarrow 6)$ sialosides, Khedri et al. extended the substrate scope to include 6FManNAc, 6FManNGc and 6FMan derivatives (Scheme 46). Yields of the corresponding sialosides were $>95 \%$ in all cases, which is comparable or better than that observed with the natural substrates. ${ }^{90}$ 

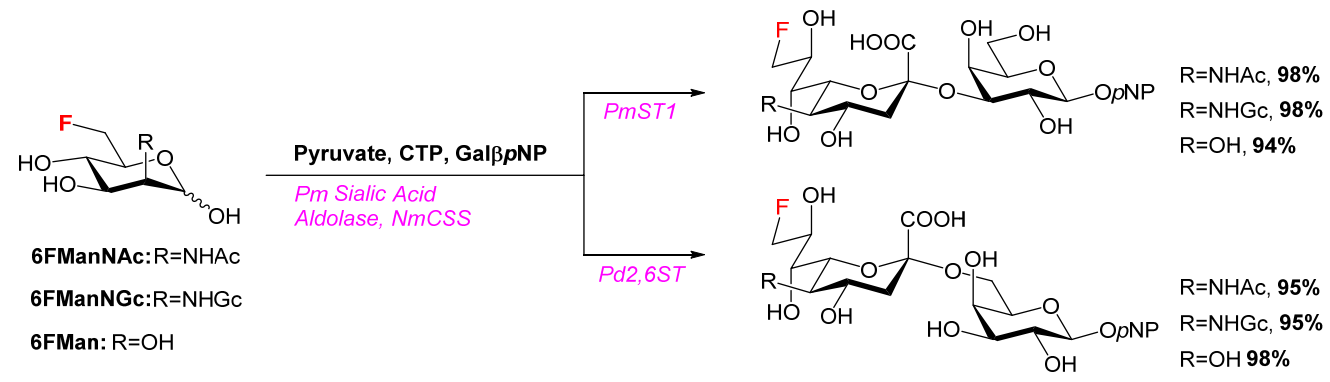

Scheme 46 The one-pot, three enzyme synthesis of sialosides containing 9F modified sialic acid derivatives. ${ }^{90}$

Kajihara et al. presented a strategy to introduce 13-C labelling at the C-3 of modified Neu5Ac residues, taking advantage of the established synthetic routes used to obtain fluorinated Neu5Ac derivatives from Neu5Ac using selective protection and deprotection. This avoided the synthesis of the fluorinated ManNAc analogues, of which the authors argue the synthetic chemistry of which has been less explored. Thus, two aldolase reactions were performed, the first, coupled with pyruvate removal by lactate dehydrogenase, promoted the degradation of the fluorinated Neu5Ac derivative to the hexose (Scheme 47). After conversion to the hexose and switching off of the cofactor regeneration cycle, the second aldose reaction with labelled pyruvate resulted in the labelled Neu5Ac analogue. Using this two-step, one-pot reaction, $9 \mathrm{~F}\left[3-{ }^{13} \mathrm{C}\right] \mathrm{Neu} 5 \mathrm{Ac}$ was synthesised in milligram scales (62\% yield) with $96 \%{ }^{13} \mathrm{C}$ labelling incorporated. ${ }^{91}$ Subsequent chemical conversion of $9 \mathrm{~F}\left[3-{ }^{13} \mathrm{C}\right] \mathrm{Neu} 5 \mathrm{Ac}$ into CMP-9F $\left[3-{ }^{13} \mathrm{C}\right] \mathrm{Neu} 5 \mathrm{Ac}$ allowed for $\alpha-(2 \rightarrow 6)$ sialyl transfer (bacterial) onto a glycosylated protein. ${ }^{92}$

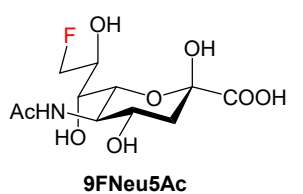

chemical synthesis

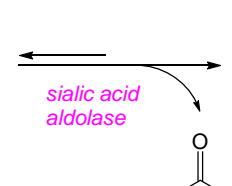

$\stackrel{\mathrm{NOOH}}{\mathrm{COOH}}^{\mathrm{O}}$

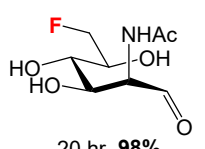

$20 \mathrm{hr}, \mathbf{9 8 \%}$

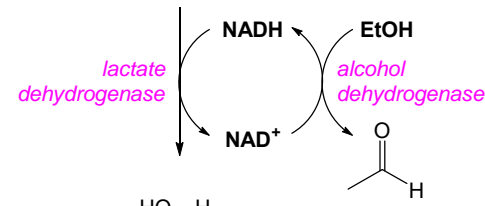

$\mathrm{X}_{\mathrm{COOH}}^{\mathrm{HO}}$

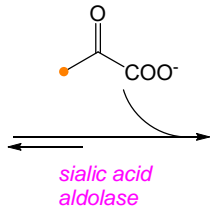

aldolase

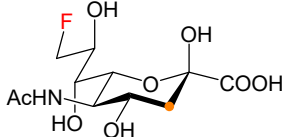

$9 \mathrm{~F}\left[3-{ }^{13} \mathrm{C}\right] \mathrm{Neu} 5 \mathrm{Ac}$

$24 \mathrm{hr}, 62 \%$

Scheme 47 The enzymatic synthesis of $9 \mathrm{~F}\left[3-{ }^{13} \mathrm{C}\right]$ Neu5Ac from $9 F N e u 5 A c$, orange dot denotes the position of the isotopic label. ${ }^{91}$ 


\section{Conclusion}

Glycosylation of fluorinated sugars using enzymatic methods has been achieved for a wide range of monofluorinated sugars and has been employed in the synthesis of many fluorinated oligosaccharides.

Monofluorinated monosaccharides including galactose, glucose and sialic acid have been successfully utilised as donors and acceptors for glycosylation, for the production of a diverse range of fluorinated disaccharide and trisaccharide targets, and phosphorylated di- and tetrafluorinated galactose donors have been enzymatically synthesised, although not yet successfully used in enzymatic glycosylations. The incorporation of more heavily fluorinated monosaccharides into glycans through glycosylation remains challenging by enzymatic means, however enzymatic glycosylations to date have relied mainly on the use of natural enzymes, and the future development of engineered enzymes able to accept a wider range of unnatural substrates could provide new routes to glycans with more complicated fluorination patterns. Enzyme reaction rates using fluorinated sugars are often low in comparison to the natural substrates, but it has been demonstrated that where an unnatural sugar is able to be accepted by the enzyme, even sugars initially considered competitive inhibitors of the enzyme can be enzymatically transformed with careful optimisation of reaction conditions. One limitation of enzymatic synthesis is the low commercial availability of glyco-enzymes, with enzyme supply often reliant on transfers of enzymes, plasmids or expressed cells between researchers and in-house enzyme production, which restricts the uptake of these methods by chemists, who may choose a more familiar chemical glycosylation route over an enzymatic route that requires production of the specific enzyme for the transformation. The next generation of fluorinated glycans are likely to require a combination of chemical and enzymatic transformations to achieve more diverse fluorination patterns.

\section{Conflicts of Interests}

There are no conflicts to declare.

\section{Acknowledgements}

This work has been funded by the Industrial Biotechnology Catalyst (Innovate UK, BBSRC, EPSRC) to support the translation, development and commercialisation of innovative Industrial Biotechnology processes (BB/M028941/1 and BB/M028879/1). 


\section{References}

1. Tyrikos-Ergas, T.; Fittolani, G.; Seeberger, P. H.; Delbianco, M., Structural Studies Using Unnatural Oligosaccharides: Toward Sugar Foldamers. Biomacromolecules 2019, 21 , 18-29.

2. Ardá, A.; Jiménez-Barbero, J., The recognition of glycans by protein receptors. Insights from NMR spectroscopy. Chemical Communications 2018, 54 (38), 4761-4769.

3. Bilska-Markowska, M.; Szwajca, A.; Marciniak, B., Design, properties and applications of fluorinated and fluoroalkylated $\mathrm{N}$-containing monosaccharides and their analogues. Journal of Fluorine Chemistry 2019, 227, 109364.

4. $\quad$ Cavaliere, A.; Probst, K. C.; Westwell, A. D.; Slusarczyk, M., Fluorinated nucleosides as an important class of anticancer and antiviral agents. Future Medicinal Chemistry 2017, 9 (15), 1809-1833.

5. $\quad$ van der Born, D.; Pees, A.; Poot, A. J.; Orru, R. V. A.; Windhorst, A. D.; Vugts, D. J., Fluorine-18 labelled building blocks for PET tracer synthesis. Chemical Society Reviews 2017, 46 (15), 4709-4773.

6. Tysoe, C.; Withers, S. G., Fluorinated Mechanism-Based Inhibitors: Common Themes and Recent Developments. Current Topics in Medicinal Chemistry 2014, 14 (7), 865-874.

7. Street, I. P.; Armstrong, C. R.; Withers, S. G., Hydrogen bonding and specificity. Fluorodeoxy sugars as probes of hydrogen bonding in the glycogen phosphorylase-glucose complex. Biochemistry 1986, 25 (20), 6021-6027.

8. Burkart, M. D.; Vincent, S. P.; Düffels, A.; Murray, B. W.; Ley, S. V.; Wong, C.-H., Chemo-Enzymatic Synthesis of Fluorinated Sugar Nucleotide: Useful Mechanistic Probes for Glycosyltransferases. Bioorganic \& Medicinal Chemistry 2000, 8, 1937-1946.

9. Matei, E.; André, S.; Glinschert, A.; Infantino, A. S.; Oscarson, S.; Gabius, H.-J.; Gronenborn, A. M., Fluorinated Carbohydrates as Lectin Ligands: Dissecting GlycanCyanovirin Interactions by Using 19F NMR Spectroscopy. Chemistry - A European Journal 2013, 19 (17), 5364-5374.

10. Rajendran, J. G.; Wilson, D. C.; Conrad, E. U.; Peterson, L. M.; Bruckner, J. D.; Rasey, J. S.; Chin, L. K.; Hofstrand, P. D.; Grierson, J. R.; Eary, J. F.; Krohn, K. A., [18F]FMISO and [18F]FDG PET imaging in soft tissue sarcomas: correlation of hypoxia, metabolism and VEGF expression. European Journal of Nuclear Medicine and Molecular Imaging 2003, 30 (5), 695704.

11. Prante, O.; Hamacher, K.; Coenen, H. H., Chemoenzymatic n.c.a synthesis of the coenzyme UDP-2-deoxy-2-(18F)fluoro-a-D-glucopyranose as substrate of glycosyltransferases. Journal of Labelled Compounds and Radiopharmaceuticals 2007, 50, 55-63.

12. Williams, S. J.; Withers, S. G., Glycosyl fluorides in enzymatic reactions. Carbohydrate Research 2000, 327 (1), 27-46.

13. Johannes, M.; Reindl, M.; Gerlitzki, B.; Schmitt, E.; Hoffmann-Röder, A., Synthesis and biological evaluation of a novel MUC1 glycopeptide conjugate vaccine candidate comprising a 4'-deoxy-4'-fluoro-Thomsen-Friedenreich epitope. Beilstein Journal of Organic Chemistry 2015, 11, 155-161.

14. Li, W.; McArthur, J. B.; Chen, X., Strategies for chemoenzymatic synthesis of carbohydrates. Carbohydrate Research 2019, 472, 86-97.

15. Uhrig, M. L.; Lantaño, B.; Postigo, A., Synthetic strategies for fluorination of carbohydrates. Organic \& Biomolecular Chemistry 2019, 17 (21), 5173-5189. 
16. Cheng, Y.; Guo, A.-L.; Guo, D.-S., Recent Progress in Synthesis and Applications of Fluorinated Carbohydrates. Current Organic Chemistry 2010, 14 (9), 977-999.

17. Withers, S. G.; Street, I. P.; Rettig, S. J., The preferred conformation of 2-fluoro-2deoxy $\beta$-D-mannopyranosyl fluoride. An X-ray crystallographic and 2-dimensional proton nuclear magnetic resonance study. Canadian Journal of Chemistry 1986, 64 (2), 232-236. 18. Linclau, B.; Golten, S.; Light, M.; Sebban, M.; Oulyadi, H., The conformation of tetrafluorinated methyl galactoside anomers: crystallographic and NMR studies. Carbohydrate Research 2011, 346 (9), 1129-1139.

19. Graton, J.; Wang, Z.; Brossard, A.-M.; Gonçalves Monteiro, D.; Le Questel, J.-Y.; Linclau, B., An unexpected and significantly lower hydrogen-bond-donating capacity of fluorohydrins compared to nonfluorinated alcohols. Angew Chem Int Ed Engl 2012, 51 (25), 6176-6180.

20. Graton, J.; Compain, G.; Besseau, F.; Bogdan, E.; Watts, J. M.; Mtashobya, L.; Wang, Z.; Weymouth-Wilson, A.; Galland, N.; Le Questel, J.-Y.; Linclau, B., Influence of Alcohol $\beta$ Fluorination on Hydrogen-Bond Acidity of Conformationally Flexible Substrates. Chemistry A European Journal 2017, 23 (12), 2811-2819.

21. Hayashi, T.; Murray, B. W.; Wang, R.; Wong, C.-H., A Chemoenymatic Synthesis of UDP-(2-deoxy-2-fluoro)-galactose and Evaluation of its Interaction with Galactosyltransferase. Bioorganic \& Medicinal Chemistry 1997, 5, 497-500.

22. Errey, J. C.; Mukhopadhyay, B.; Kartha, K. P. R.; Field, R. A., Flexible enzymatic and chemo-enzymatic approaches to a broad range of uridine-diphospho-sugars. Chemical Communications 2004, (23), 2706-2707.

23. Danby, P. M.; Withers, S. G., Advances in Enzymatic Glycoside Synthesis. ACS Chemical Biology 2016, 11 (7), 1784-1794.

24. Frasch, A. C. C., Functional Diversity in the Trans-sialidase and Mucin Families in Trypanosoma cruzi. Parasitology Today 2000, 16 (7), 282-286.

25. Kent, P. W.; Wright, J. R., Fluorocarbohydrates. Part XXIV. Chemical and Enzymatic Reduction and Phosphorylation of 6-deoxy-6-fluoro-a-D-galactose. Carbohydrate Research 1972, 22 (1), 193-200.

26. Thomas, P.; Bessell, E. M.; Westwood, J. H., The use of deoxyfluoro-Dgalactopyranoses in a study of yeast galactokinase specificity. Biochemical Journal 1974, 139 (3), 661-664.

27. Kean, E., CMP-sialic acid synthetase of the nucleus. Biochimica et Biophysica Acta (BBA) - General Subjects 2004, 1673 (1-2), 56-65.

28. Barlow, J. N.; Blanchard, J. S., Enzymatic synthesis of UDP-(3-deoxy-3-fluoro)-Dgalactose and UDP-(2-deoxy-2-fluoro)-D-galactose and substrate activity with UDPgalactopyranose mutase. Carbohydrate Research 2000, 328 (4), 473-480.

29. Bessell, E. M.; Foster, A. B.; Westwood, J. H., The use of deoxyfluoro-Dglucopyranoses and related compounds in a study of yeast hexokinase specificity. Biochemical Journal 1972, 128 (2), 199-204.

30. Nishimura, S.-I.; Nagahori, N.; Hamamoto, T.; Okuyama, K.; Noguchi, T. Sugar chain containing 4-position halogenated galactose and application thereof. 2012.

31. Yang, J.; Liu, L.; Thorson, J. S., Structure-Based Enhancement of the First Anomeric Glucokinase. ChemBioChem 2004, 5 (7), 992-996.

32. Timmons, S. C.; Hui, J. P. M.; Pearson, J. L.; Peltier, P.; Daniellou, R.; Nugier-Chauvin, C.; Soo, E. C.; Syvitski, R. T.; Ferrières, V.; Jakeman, D. L., Enzyme-Catalyzed Synthesis of Furanosyl Nucleotides. Organic Letters 2008, 10 (2), 161-163. 
33. Jakeman, D. L.; Young, J. L.; Huestis, M. P.; Peltier, P.; Daniellou, R.; Nugier-Chauvin, C.; Ferrières, V., Engineering Ribonucleoside Triphosphate Specificity in a Thymidylyltransferase. Biochemistry 2008, 47 (33), 8719-8725.

34. Loch, N.; Geilen, C. C.; Spörndle, I.; Oberdorfer, F.; Keppler, D.; Tauber, R.; Reutter, W., 2-Deoxy-2-fluoro-D-galactose protein N-glycosylation. FEBS Letters 1991, 294 (3), 217 220.

35. Kodama, H.; Kajihara, Y.; Endo, T.; Hashimoto, H., Synthesis of UDP-6-deoxy- and -6fluoro-D-galactoses and their enzymatic glycosyl transfer to mono- and biantennary carbohydrate chains. Tetrahedron Letters 1993, 34 (40), 6419-6422.

36. Schengrund, C.-L.; Kováč, P., UDP-6-deoxy-6-fluoro- $\alpha$-d-galactose binds to two different galactosyltransferases, but neither can effectively catalyze transfer of the modified galactose to the appropriate acceptor. Carbohydrate Research 1999, 319 (1-4), 24-28.

37. Yu, H.; Thon, V.; Lau, K.; Cai, L.; Chen, Y.; Mu, S.; Li, Y.; Wang, P. G.; Chen, X., Highly efficient chemoenzymatic synthesis of $\beta 1-3$-linked galactosides. Chemical Communications 2010, 46 (40), 7507-7509.

38. Yan, J.; Chen, X.; Wang, F.; Cao, H., Chemoenzymatic synthesis of mono- and difluorinated Thomsen-Friedenreich $(T)$ antigens and their sialylated derivatives. Organic \& Biomolecular Chemistry 2013, 11 (5), 842-848.

39. Li, L.; Liu, Y.; Li, T.; Wang, W.; Yu, Z.; Ma, C.; Qu, J.; Zhao, W.; Chen, X.; Wang, P. G., Efficient chemoenzymatic synthesis of novel galacto-N-biose derivatives and their sialylated forms. Chemical Communications 2015, 51 (51), 10310-10313.

40. Euzen, R.; Lopez, G.; Nugier-Chauvin, C.; Ferrières, V.; Plusquellec, D.; Rémond, C.; O'Donohue, M., A Chemoenzymatic Approach for the Synthesis of Unnatural Disaccharides Containing D-Galacto- or D-Fucofuranosides. European Journal of Organic Chemistry 2005, 2005 (22), 4860-4869.

41. Chlubnová, I.; Králová, B.; Dvořáková, H.; Spiwok, V.; Filipp, D.; Nugier-Chauvin, C.; Daniellou, R.; Ferrières, V., Biocatalyzed synthesis of difuranosides and their ability to trigger production of TNF- $\alpha$. Bioorganic \& Medicinal Chemistry Letters 2016, 26 (6), 1550-1553.

42. Watkins, W. M., Chapter 5 Biosynthesis 5. Molecular Basis of Antigenic Specificity in the ABO, $\mathrm{H}$ and Lewis Blood-Group Systems. In New Comprehensive Biochemistry, Montreuil, J.; Vliegenthart, J. F. G.; Schachter, H., Eds. Elsevier: 1995; Vol. 29, pp 313-390. 43. Lowary, T. L.; Hindsgaul, O., Recognition of synthetic deoxy and deoxyfluoro analogs of the acceptor $\alpha$-I-Fucp-( $1 \rightarrow 2)-\beta$-d-Galp-OR by the blood-group A and B gene-specified glycosyltransferases. Carbohydrate Research 1993, 249 (1), 163-195.

44. Kasuya, M. C.; Ito, A.; Hatanaka, K., Simple and convenient synthesis of a fluorinated GM4 analogue. Journal of Fluorine Chemistry 2007, 128 (5), 562-565.

45. Allman, S. A.; Jensen, H. H.; Vijayakrishnan, B.; Garnett, J. A.; Leon, E.; Liu, Y.; Anthony, D. C.; Sibson, N. R.; Feizi, T.; Matthews, S.; Davis, B. G., Potent Fluorooligosaccharide Probes of Adhesion in Toxoplasmosis. ChemBioChem 2009, 10 (15), 25222529.

46. Van Dorst, J. A. L. M.; Tikkanen, J. M.; Krezdorn, C. H.; Streiff, M. B.; Berger, E. C.; Van Kuik, J. A.; Kamerling, J. P.; Vliegenthart, J. F. G., Exploring the substrate specificities of the a2,6- and a-2,3-sialyltransferases using synthetic acceptor analogues. European Journal of Biochemistry 1996, 242, 674-681.

47. Unverzagt, C.; Kunz, H.; Paulson, J. C., High-efficiency synthesis of sialyloligosaccharides and sialoglycopeptides. Journal of the American Chemical Society 1990, 112 (25), 9308-9309. 
48. Caputi, L.; Rejzek, M.; Louveau, T.; O'Neill, E. C.; Hill, L.; Osbourn, A.; Field, R. A., A one-pot enzymatic approach to the O-fluoroglucoside of $\mathrm{N}$-methylanthranilate. Bioorganic \& Medicinal Chemistry 2013, 21, 4762-4767.

49. Gantt, R. W.; Peltier-Pain, P.; Cournoyer, W. J.; Thorson, J. S., Using simple donors to drive the equilibria of glycosyltransferase-catalyzed reactions. Nature Chemical Biology 2011, 7, 685-691.

50. Zhu, J.-S.; McCormick, N. E.; Timmons, S. C.; Jakeman, D. L., Synthesis of $\alpha-$ Deoxymono and Difluorohexopyranosyl 1-Phosphates and Kinetic Evaluation with Thymidylyl- and Guanidylyltransferases. The Journal of Organic Chemistry 2016, 81, 88168825.

51. Jiang, J.; Biggins, J. B.; Thorson, J. S., A General Enzymatic Method for the Synthesis of Natural and "Unnatural" UDP- and TDP-Nucleotide Sugars. Journal of the American Chemical Society 2000, 122 (28), 6803-6804.

52. Lee, S. S.; Hong, S. Y.; Errey, J. C.; Izumi, A.; Davies, G. J.; Davis, B. G., Mechanistic evidence for a front-side, SNi-type reaction in a retaining glycosyltransferase. Nat Chem Biol 2011, 7 (9), 631-638.

53. Backus, K. M.; Boshoff, H. I.; Barry, C. S.; Boutureira, O.; Patel, M. K.; D'Hooge, F.; Lee, S. S.; Via, L. E.; Tahlan, K.; Barry, C. E.; Davis, B. G., Uptake of unnatural trehalose analogs as a reporter for Mycobacterium tuberculosis. Nat Chem Biol 2011, 7 (4), 228-235. 54. Rundell, S. R.; Wagar, Z. L.; Meints, L. M.; Olson, C. D.; O'Neill, M. K.; Piligian, B. F.; Poston, A. W.; Hood, R. J.; Woodruff, P. J.; Swarts, B. M., Deoxyfluoro-d-trehalose (FDTre) analogues as potential PET probes for imaging mycobacterial infection. Organic \& Biomolecular Chemistry 2016, 14 (36), 8598-8609.

55. Mackenzie, L. F.; Wang, Q.; Warren, R. A. J.; Withers, S. G., Glycosynthases: Mutant Glycosidases for Oligosaccharide Synthesis. Journal of the American Chemical Society 1998, 120 (22), 5583-5584.

56. André, C.; Spangenberg, P.; Gentil, E.; Rabiller, C., In situ 19F NMR spectroscopy study of enzymatic transglycosylation reactions using $\alpha$-d-aldohexopyranosyl fluorides as donors and acceptors. Tetrahedron: Asymmetry 2001, 12 (5), 779-783.

57. Tantanarat, K.; Rejzek, M.; O’Neill, E.; Ruzanski, C.; Hill, L.; Fairhurst, S. A.; Limpaseni, T.; Field, R. A., An expedient enzymatic route to isomeric 2-, 3- and 6-monodeoxymonofluoro-maltose derivatives. Carbohydrate Research 2012, 358, 12-18.

58. Feng, F.; Okuyama, K.; Niikura, K.; Ohta, T.; Sadamoto, R.; Monde, K.; Noguchi, T.; Nishimura, S.-I., Chemo-enzymatic synthesis of fluorinated 2-N-acetamidosugar nucleotides using UDP-GICNAc pyrophosphorylase. Organic \& Biomolecular Chemistry 2004, 2 (11), 1617-1623.

59. Nishimura, S.-I.; Hato, M.; Hyugaji, S.; Feng, F.; Amano, M., Glycomics for Drug Discovery: Metabolic Pertubation in Androgen-Independent Prostate Cancer Cells Induced by Unnatural Hexosamine Mimics. Angewandte Chemie International Edition 2012, 51, 3386-3390.

60. Hartman, M. C. T.; Coward, J. K., Synthesis of 5-Fluoro N-Acetylglucosamine Glycosides and Pyrophosphates vai Epoxide Fluoridolysis: Versatile Reagents for the Study of Glycoconjugate Biochemistry. Journal of the American Chemical Society 2002, 124, 10036-10053.

61. Hartman, M. C. T.; Jiang, S.; Rush, J. S.; Waechter, C. J.; Coward, J. K., Glycosyltransferase Mechanisms: Impact of a 5-Fluoro Substituent in Acceptor and Donor Substrates on Catalysis. Biochemistry 2007, 46, 11630-11638. 
62. Frantom, P. A.; Coward, J. K.; Blanchard, J. S., UDP-(5F)-GIcNAc acts as a slow binding inhibitor of MShA, a retaining glycosyltransferase. Journal of the American Chemical Society 2010, 132, 6626-6627.

63. Sala, R. F.; MacKinnon, S. L.; Palcic, M. M.; Tanner, M. E., UDP-Ntrifluoroacetylglucosamine as an alternative substrate in $\mathrm{N}$-acetylglucosaminyltransferase reactions. Carbohydrate Research 1998, 306 (1), 127-136.

64. Chen, Y.; Li, Y.; Yu, H.; Sugiarto, G.; Thon, V.; Hwang, J.; Ding, L.; Hie, L.; Chen, X., Tailored Design and Synthesis of Heparan Sulfate Oligosaccharide Analogues Using Sequential One-Pot Multienzyme Systems. Angewandte Chemie International Edition 2013, 52 (45), 11852-11856.

65. Li, Y.; Yu, H.; Thon, V.; Chen, Y.; Muthana, M. M.; Qu, J.; Hie, L.; Chen, X., Donor substrate promiscuity of the $\mathrm{N}$-acetylglucosaminyltransferase activities of Pasteurella multocida heparosan synthase 2 (PmHS2) and Escherichia coli K5 KfiA. Applied Microbiology and Biotechnology 2014, 98 (3), 1127-1134.

66. Chen, Y.; Thon, V.; Li, Y.; Yu, H.; Ding, L.; Lau, K.; Qu, J.; Hie, L.; Chen, X., One-pot three-enzyme synthesis of UDP-GIcNAc derivatives. Chemical Communications 2011, 47 (38), 10815-10817.

67. Zhang, X.; Lin, L.; Huang, H.; Linhardt, R. J., Chemoenzymatic Synthesis of Glycosaminoglycans. Accounts of Chemical Research 2019.

68. Errey, J. C.; Mann, M. C.; Fairhurst, S. A.; Hill, L.; McNeil, M. R.; Naismith, J. H.; Percy, J. M.; Whitfield, C.; Field, R. A., Sugar nucleotide recognition by Klebsiella pneumoniaeUDPd-galactopyranose mutase: Fluorinated substrates, kinetics and equilibria. Organic \& Biomolecular Chemistry 2009, 7 (5), 1009-1016.

69. Baisch, G.; Öhrlein, R.; Katopodis, A.; Streiff, M.; Kolbinger, F., Synthetic potential of cloned fucosyl-transferase III and VI. Bioorganic \& Medicinal Chemistry Letters 1997, 7, 2447-2450.

70. Lairson, L. L.; Henrissat, B.; Davies, G. J.; Withers, S. G., Glycosyltransferases: Structures, Functions and Mechanisms. Annual Review of Biochemistry 2008, 77, 521-555.

71. Murray, B. W.; Wittmann, V.; Burkart, M. D.; Hung, S.-C.; Wong, C.-H., Mechanism of

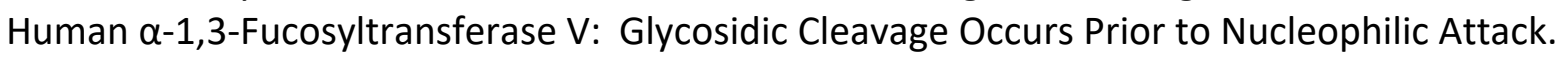
Biochemistry 1997, 36 (4), 823-831.

72. Wang, W.; Hu, T.; Frantom, P. A.; Zheng, T.; Gerwe, B.; del Amo, D. S.; Garret, S.; Seidel, R. D.; Wu, P., Chemoenzymatic synthesis of GDP-l-fucose and the Lewis X glycan derivatives. Proceedings of the National Academy of Sciences 2009, 106 (38), 16096-16101. 73. Lee, H.-Y.; Chen, C.-Y.; Tsai, T.-I.; Li, S.-T.; Lin, K.-H.; Cheng, Y.-Y.; Ren, C.-T.; Cheng, T.J. R.; Wu, C.-Y.; Wong, C.-H., Immunogenicity Study of Globo H Analogues with Modification at the Reducing or Nonreducing End of the Tumor Antigen. Journal of the American Chemical Society 2014, 136 (48), 16844-16853.

74. Watts, A. G.; Withers, S. G., The synthesis of some mechanistic probes for sialic acid processing enzymes and the labeling of a sialidase from Trypanosoma rangeli. Canadian Journal of Chemistry 2004, 82 (11), 1581-1588.

75. Li, Y.; Yu, H.; Cao, H.; Lau, K.; Muthana, S.; Tiwari, V. K.; Son, B.; Chen, X., Pasteurella multocida sialic acid aldolase: a promising biocatalyst. Applied Microbiology and Biotechnology 2008, 79 (6), 963-970.

76. Chokhawala, H. A.; Cao, H.; Yu, H.; Chen, X., Enzymatic Synthesis of Fluorinated Mechanistic Probes for Sialidases and Sialyltransferases. Journal of the American Chemical Society 2007, 129 (35), 10630-10631. 
77. Petrie III, C. R.; Sharma, M.; SImmons, O. D.; Korytnyk, W., Synthesis of analogs of Nacetylneuraminic acid and their effect on CMP-sialate synthase. Carbohydrate Research 1989, 186, 326-334.

78. Burkart, M. D.; Vincent, S. P.; Wong, C.-H., An efficient synthesis of CMP-3fluoroneuraminic acid. Chemical Communications 1999, 1525-1526.

79. Heise, T.; Pijnenborg, J. F. A.; Büll, C.; Van Hilten, N.; Kers-Rebel, E. D.; Balneger, N.; Elferink, H.; Adema, G. J.; Boltje, T. J., Potent Metabolic Sialylation Inhibitors Based on C-5Modified Fluorinated Sialic Acids. Journal of Medicinal Chemistry 2019, 62 (2), 1014-1021. 80. Chiu, C. P. C.; Watts, A. G.; Lairson, L. L.; Gilbert, M.; Lim, D.; Wakarchuk, W. W.; Withers, S. G.; Strynadka, N. C. J., Structural analysis of the sialyltransferase Cstll from Campylobacter jejuni in complex with a substrate analog. Nat Struct Mol Biol 2004, 11 (2), 163-170.

81. Watts, A. G.; Damager, I.; Amaya, M. L.; Buschiazzo, A.; Alzari, P. M.; Frasch, A. C.; Withers, S. G., Trypanosoma cruzi Trans-sialidase operates through a covalent sialyl-enzyme intermediate: Tyrosine is the Catalytic nucleophile. Journal of the American Chemical Society 2003, 125, 7532-7533.

82. Liu, J. L. C.; Shen, G. J.; Ichikawa, Y.; Rutan, J. F.; Zapata, G.; Vann, W. F.; Wong, C. H., Overproduction of CMP-sialic acid synthetase for organic synthesis. Journal of the American Chemical Society 1992, 114 (10), 3901-3910.

83. Cao, H.; Li, Y.; Lau, K.; Muthana, S.; Yu, H.; Cheng, J.; Chokhawala, H. A.; Sugiarto, G.; Zhang, L.; Chen, X., Sialidase substrate specificity studies using chemoenzymatically synthesized sialosides containing C5-modified sialic acids. Organic \& Biomolecular Chemistry 2009, 7 (24), 5137-5145.

84. Hartlieb, S.; Günzel, A.; Gerardy-Schahn, R.; Münster-Kühnel, A. K.; Kirschning, A.; Dräger, G., Chemoenzymatic synthesis of CMP-N-acetyl-7-fluoro-deoxy-neuraminic acid. Carbohydrate Research 2008, 343, 2075-2082.

85. Khedri, Z.; Li, Y.; Muthana, S.; Muthana, M. M.; Hsiao, C.-W.; Yu, H.; Chen, X., Chemoenzymatic synthesis of sialosides containing C7-modified sialic acids and thier application in sialidase substrate specifity studies. Carbohydrate Research 2014, 389, 100111.

86. Morley, T. J.; Withers, S. G., Chemoenzymatic Synthesis and Enzymatic Analysis of 8Modified Cytidine Monophosphate-Sialic Acid and Sialyl Lactose Derivatives. Journal of the American Chemical Society 2010, 132 (27), 9430-9437.

87. Yu, H.; Chokhawala, H.; Karpel, R.; Yu, H.; Wu, B.; Zhang, J.; Zhang, Y.; Jia, Q.; Chen, X., A Multifunctional Pasteurella multocida Sialyltransferase: A Powerful Tool for the Synthesis of Sialoside Libraries. Journal of the American Chemical Society 2005, 127 (50), 17618-17619.

88. Petrie III, C. R.; Korytnyk, W., A High-Performance Liquid Chromatography Method for the Assay of Cytidine Monophosphate-Sialic Acid Synthetase. Analytical Biochemistry 1983, 131, 153-159.

89. Conradt, H. S.; Bünsch, A.; Brossmer, R., Preparation of 9-fluoro-9-deoxy-N-[214C]acetylneuraminic acid. Activation and transfer onto asialo-a1-acid glycoprotein. FEBS Letters 1984, 170 (2), 295-300.

90. Khedri, Z.; Muthana, M. M.; Li, Y.; Muthana, S.; Yu, H.; Cao, H.; Chen, X., Probe sialidase substrate specificity using chemoenzymatically synthesised sialosides containing C9-modified sialic acid. Chemical Communications 2012, 48, 3357-3359. 
91. Miyazaki, T.; Sato, H.; Sakakibara, T.; Kajihara, Y., An Approach to the Precise Chemoenzymatic Synthesis of 13C-Labeled Sialyloligosaccharide on an Intact Glycoprotein: A Novel One-Pot [3-13C]-Labeling Method for Sialic Acid Analogues by Control of the Reversible Aldolase Reaction, Enzymatic Synthesis of [3-13C]-NeuAc- $\alpha-(2 \rightarrow 3)-[U-13 C]-G a l-\beta-$ $(1 \rightarrow 4)$-GlcNAc- $\beta$ - Sequence onto Glycoprotein, and Its Conformational Analysis by Developed NMR Techniques. Journal of the American Chemical Society 2000, 122 (24), 56785694.

92. Miyazaki, T.; Sakakibara, T.; Sato, H.; Kajihara, Y., Chemoenzymatic Synthesis of the 9-Deoxy- 9-fluoro-[3-13C]-NeuAc- $\alpha-(2 \rightarrow 6)-[U-13 C]-G a l-\beta$ - Sequence on an Intact Glycoprotein. Journal of the American Chemical Society 1999, 121 (6), 1411-1412. 